\title{
The Dynamics of Viral Marketing *
}

\author{
Jure Leskovec \\ Machine Learning Department, Carnegie Mellon University, Pittsburgh, PA \\ Lada A. Adamic \\ School of Information, University of Michigan, Ann Arbor, MI \\ Bernardo A. Huberman \\ HP Labs, Palo Alto, CA 94304
}

February 2, 2008

\begin{abstract}
We present an analysis of a person-to-person recommendation network, consisting of 4 million people who made 16 million recommendations on half a million products. We observe the propagation of recommendations and the cascade sizes, which we explain by a simple stochastic model. We analyze how user behavior varies within user communities defined by a recommendation network. Product purchases follow a 'long tail' where a significant share of purchases belongs to rarely sold items. We establish how the recommendation network grows over time and how effective it is from the viewpoint of the sender and receiver of the recommendations. While on average recommendations are not very effective at inducing purchases and do not spread very far, we present a model that successfully identifies communities, product and pricing categories for which viral marketing seems to be very effective.
\end{abstract}

\section{Introduction}

With consumers showing increasing resistance to traditional forms of advertising such as TV or newspaper ads, marketers have turned to alternate strategies, including viral marketing. Viral marketing exploits existing social networks by encouraging customers to share product information with their friends. Previously, a few in depth studies have shown that social networks affect the adoption of individual innovations and products (for a review see Rog95 or [SS98]). But until recently it has been difficult to measure how influential person-to-person recommendations actually are over a wide range of products. Moreover, Subramani and Rajagopalan SR03 noted that "there needs to be a greater understanding of the contexts in which viral marketing strategy works and the characteristics of products and services for which it is most

\footnotetext{
${ }^{*}$ This work also appears in: Leskovec, J., Adamic, L. A., and Huberman, B. A. 2007. The dynamics of viral marketing. ACM Transactions on the Web, 1, 1 (May 2007).
} 
effective. This is particularly important because the inappropriate use of viral marketing can be counterproductive by creating unfavorable attitudes towards products. What is missing is an analysis of viral marketing that highlights systematic patterns in the nature of knowledge-sharing and persuasion by influencers and responses by recipients in online social networks."

Here we were able to in detail study the above mentioned problem. We were able to directly measure and model the effectiveness of recommendations by studying one online retailer's incentivised viral marketing program. The website gave discounts to customers recommending any of its products to others, and then tracked the resulting purchases and additional recommendations.

Although word of mouth can be a powerful factor influencing purchasing decisions, it can be tricky for advertisers to tap into. Some services used by individuals to communicate are natural candidates for viral marketing, because the product can be observed or advertised as part of the communication. Email services such as Hotmail and Yahoo had very fast adoption curves because every email sent through them contained an advertisement for the service and because they were free. Hotmail spent a mere $\$ 50,000$ on traditional marketing and still grew from zero to 12 million users in 18 months Jur00. The Hotmail user base grew faster than any media company in history - faster than CNN, faster than AOL, even faster than Seinfeld's audience. By mid-2000, Hotmail had over 66 million users with 270,000 new accounts being established each day Bro98. Google's Gmail also captured a significant part of market share in spite of the fact that the only way to sign up for the service was through a referral.

Most products cannot be advertised in such a direct way. At the same time the choice of products available to consumers has increased manyfold thanks to online retailers who can supply a much wider variety of products than traditional brick-andmortar stores. Not only is the variety of products larger, but one observes a 'fat tail' phenomenon, where a large fraction of purchases are of relatively obscure items. On Amazon.com, somewhere between 20 to 40 percent of unit sales fall outside of its top 100,000 ranked products BHS03. Rhapsody, a streaming-music service, streams more tracks outside than inside its top 10,000 tunes [Ano05. Some argue that the presence of the long tail indicates that niche products with low sales are contributing significantly to overall sales online.

We find that product purchases that result from recommendations are not far from the usual 80-20 rule. The rule states that the top twenty percent of the products account for 80 percent of the sales. In our case the top $20 \%$ of the products contribute to about half the sales.

Effectively advertising these niche products using traditional advertising approaches is impractical. Therefore using more targeted marketing approaches is advantageous both to the merchant and the consumer, who would benefit from learning about new products.

The problem is partly addressed by the advent of online product and merchant reviews, both at retail sites such as EBay and Amazon, and specialized product comparison sites such as Epinions and CNET. Of further help to the consumer are collaborative filtering recommendations of the form "people who bought $x$ also bought $y$ " feature LSY03. These refinements help consumers discover new products and receive more accurate evaluations, but they cannot completely substitute personalized 
recommendations that one receives from a friend or relative. It is human nature to be more interested in what a friend buys than what an anonymous person buys, to be more likely to trust their opinion, and to be more influenced by their actions. As one would expect our friends are also acquainted with our needs and tastes, and can make appropriate recommendations. A Lucid Marketing survey found that $68 \%$ of individuals consulted friends and relatives before purchasing home electronics - more than the half who used search engines to find product information [Bur03].

In our study we are able to directly observe the effectiveness of person to person word of mouth advertising for hundreds of thousands of products for the first time. We find that most recommendation chains do not grow very large, often terminating with the initial purchase of a product. However, occasionally a product will propagate through a very active recommendation network. We propose a simple stochastic model that seems to explain the propagation of recommendations.

Moreover, the characteristics of recommendation networks influence the purchase patterns of their members. For example, individuals' likelihood of purchasing a product initially increases as they receive additional recommendations for it, but a saturation point is quickly reached. Interestingly, as more recommendations are sent between the same two individuals, the likelihood that they will be heeded decreases.

We find that communities (automatically found by graph theoretic community finding algorithm) were usually centered around a product group, such as books, music, or DVDs, but almost all of them shared recommendations for all types of products. We also find patterns of homophily, the tendency of like to associate with like, with communities of customers recommending types of products reflecting their common interests.

We propose models to identify products for which viral marketing is effective: We find that the category and price of product plays a role, with recommendations of expensive products of interest to small, well connected communities resulting in a purchase more often. We also observe patterns in the timing of recommendations and purchases corresponding to times of day when people are likely to be shopping online or reading email.

We report on these and other findings in the following sections. We first survey the related work in section 2. We then describe the characteristics of the incentivised recommendations program and the dataset in section 3 Section 4 studies the temporal and static characteristics of the recommendation network. We investigate the propagation of recommendations and model the cascading behavior in section 5 , Next we concentrate on the various aspects of the recommendation success from the viewpoint of the sender and the recipient of the recommendation in section 6 . The timing and the time lag between the recommendations and purchases is studied in section 7 . We study network communities, product characteristics and the purchasing behavior in section 8 Last, in section 9 we present a model that relates product characteristics and the surrounding recommendation network to predict the product recommendation success. We discuss the implications of our findings and conclude in section 10 . 


\section{Related work}

Viral marketing can be thought of as a diffusion of information about the product and its adoption over the network. Primarily in social sciences there is a long history of the research on the influence of social networks on innovation and product diffusion. However, such studies have been typically limited to small networks and typically a single product or service. For example, Brown and Reingen BR87] interviewed the families of students being instructed by three piano teachers, in order to find out the network of referrals. They found that strong ties, those between family or friends, were more likely to be activated for information flow and were also more influential than weak ties Gra73 between acquaintances. Similar observations were also made by DeBruyn and Lilien in DL04 in the context of electronic referrals. They found that characteristics of the social tie influenced recipients behavior but had different effects at different stages of decision making process: tie strength facilitates awareness, perceptual affinity triggers recipients interest, and demographic similarity had a negative influence on each stage of the decision-making process.

Social networks can be composed by using various information, i.e. geographic similarity, age, similar interests and so on. Yang and Allenby [YA03] showed that the geographically defined network of consumers is more useful than the demographic network for explaining consumer behavior in purchasing Japanese cars. A recent study by Hill et al. [HPV06] found that adding network information, specifically whether a potential customer was already "talking to" an existing customer, was predictive of the chances of adoption of a new phone service option. For the customers linked to a prior customer the adoption rate of was 3-5 times greater than the baseline.

Factors that influence customers' willingness to actively share the information with others via word of mouth have also been studied. Frenzen and Nakamoto FN93. surveyed a group of people and found that the stronger the moral hazard presented by the information, the stronger the ties must be to foster information propagation. Also, the network structure and information characteristics interact when individuals form decisions about transmitting information. Bowman and Narayandas BN01] found that self-reported loyal customers were more likely to talk to others about the products when they were dissatisfied, but interestingly not more likely when they were satisfied.

In the context of the internet word-of-mouth advertising is not restricted to pairwise or small-group interactions between individuals. Rather, customers can share their experiences and opinions regarding a product with everyone. Quantitative marketing techniques have been proposed [Mon01 to describe product information flow online, and the rating of products and merchants has been shown to effect the likelihood of an item being bought RZ02, CM06. More sophisticated online recommendation systems allow users to rate others' reviews, or directly rate other reviewers to implicitly form a trusted reviewer network that may have very little overlap with a person's actual social circle. Richardson and Domingos [RD02] used Epinions' trusted reviewer network to construct an algorithm to maximize viral marketing efficiency assuming that individuals' probability of purchasing a product depends on the opinions on the trusted peers in their network. Kempe, Kleinberg and Tardos [KKT03 have followed up on Richardson and Domingos' challenge of maximizing viral information spread by evaluating several algorithms given various models of adoption we discuss 
next.

Most of the previous research on the flow of information and influence through the networks has been done in the context of epidemiology and the spread of diseases over the network. See the works of Bailey Bai75 and Anderson and May AM02 for reviews of this area. The classical disease propagation models are based on the stages of a disease in a host: a person is first susceptible to a disease, then if she is exposed to an infectious contact she can become infected and thus infectious. After the disease ceases the person is recovered or removed. Person is then immune for some period. The immunity can also wear off and the person becomes again susceptible. Thus SIR (susceptible - infected - recovered) models diseases where a recovered person never again becomes susceptible, while SIRS (SIS, susceptible - infected - (recovered) susceptible) models population in which recovered host can become susceptible again. Given a network and a set of infected nodes the epidemic threshold is studied, i.e. conditions under which the disease will either dominate or die out. In our case SIR model would correspond to the case where a set of initially infected nodes corresponds to people that purchased a product without first receiving the recommendations. A node can purchase a product only once, and then tries to infect its neighbors with a purchase by sending out the recommendations. SIS model corresponds to less realistic case where a person can purchase a product multiple times as a result of multiple recommendations. The problem with these type of models is that they assume a known social network over which the diseases (product recommendations) are spreading and usually a single parameter which specifies the infectiousness of the disease. In our context this would mean that the whole population is equally susceptible to recommendations of a particular product.

There are numerous other models of influence spread in social networks. One of the first and most influential diffusion models was proposed by Bass Bas69. The model of product diffusion predicts the number of people who will adopt an innovation over time. It does not explicitly account for the structure of the social network but it rather assumes that the rate of adoption is a function of the current proportion of the population who have already adopted (purchased a product in our case). The diffusion equation models the cumulative proportion of adopters in the population as a function of the intrinsic adoption rate, and a measure of social contagion. The model describes an S-shaped curve, where adoption is slow at first, takes off exponentially and flattens at the end. It can effectively model word-of-mouth product diffusion at the aggregate level, but not at the level of an individual person, which is one of the topics we explore in this paper.

Diffusion models that try to model the process of adoption of an idea or a product can generally be divided into two groups:

- Threshold model Gra78 where each node in the network has a threshold $t \in$ $[0,1]$, typically drawn from some probability distribution. We also assign connection weights $w_{u, v}$ on the edges of the network. A node adopts the behavior if a sum of the connection weights of its neighbors that already adopted the behavior (purchased a product in our case) is greater than the threshold: $t \leq \sum_{\text {adopters }(u)} w_{u, v}$.

- Cascade model GLM01] where whenever a neighbor $v$ of node $u$ adopts, then node $u$ also adopts with probability $p_{u, v}$. In other words, every time a neighbor 
of $u$ purchases a product, there is a chance that $u$ will decide to purchase as well.

In the independent cascade model, Goldenberg et al. GLM01 simulated the spread of information on an artificially generated network topology that consisted both of strong ties within groups of spatially proximate nodes and weak ties between the groups. They found that weak ties were important to the rate of information diffusion. Centola and Macy CM05 modeled product adoption on small world topologies when a person's chance of adoption is dependent on having more than one contact who had previously adopted. Wu and Huberman WH04 modeled opinion formation on different network topologies, and found that if highly connected nodes were seeded with a particular opinion, this would proportionally effect the long term distribution of opinions in the network. Holme and Newman HN06 introduced a model where individuals' preferences are shaped by their social networks, but their choices of whom to include in their social network are also influenced by their preferences.

While these models address the question of how influence spreads in a network, they are based on assumed rather than measured influence effects. In contrast, our study tracks the actual diffusion of recommendations through email, allowing us to quantify the importance of factors such as the presence of highly connected individuals, or the effect of receiving recommendations from multiple contacts. Compared to previous empirical studies which tracked the adoption of a single innovation or product, our data encompasses over half a million different products, allowing us to model a product's suitability for viral marketing in terms of both the properties of the network and the product itself.

\section{The Recommendation Network}

\subsection{Recommendation program and dataset description}

Our analysis focuses on the recommendation referral program run by a large retailer. The program rules were as follows. Each time a person purchases a book, music, or a movie he or she is given the option of sending emails recommending the item to friends. The first person to purchase the same item through a referral link in the email gets a $10 \%$ discount. When this happens the sender of the recommendation receives a $10 \%$ credit on their purchase.

The following information is recorded for each recommendation

1. Sender Customer ID (shadowed)

2. Receiver Customer ID (shadowed)

3. Date of Sending

4. Purchase flag (buy-bit)

5. Purchase Date (error-prone due to asynchrony in the servers)

6. Product identifier

7. Price 
The recommendation dataset consists of 15,646,121 recommendations made among 3,943,084 distinct users. The data was collected from June 52001 to May 16 2003. In total, 548,523 products were recommended, $99 \%$ of them belonging to 4 main product groups: Books, DVDs, Music and Videos. In addition to recommendation data, we also crawled the retailer's website to obtain product categories, reviews and ratings for all products. Of the products in our data set, 5813 (1\%) were discontinued (the retailer no longer provided any information about them).

Although the data gives us a detailed and accurate view of recommendation dynamics, it does have its limitations. The only indication of the success of a recommendation is the observation of the recipient purchasing the product through the same vendor. We have no way of knowing if the person had decided instead to purchase elsewhere, borrow, or otherwise obtain the product. The delivery of the recommendation is also somewhat different from one person simply telling another about a product they enjoy, possibly in the context of a broader discussion of similar products. The recommendation is received as a form email including information about the discount program. Someone reading the email might consider it spam, or at least deem it less important than a recommendation given in the context of a conversation. The recipient may also doubt whether the friend is recommending the product because they think the recipient might enjoy it, or are simply trying to get a discount for themselves. Finally, because the recommendation takes place before the recommender receives the product, it might not be based on a direct observation of the product. Nevertheless, we believe that these recommendation networks are reflective of the nature of word of mouth advertising, and give us key insights into the influence of social networks on purchasing decisions.

\subsection{Identifying successful recommendations}

For each recommendation, the dataset includes information about the recommended product, sender and received or the recommendation, and most importantly, the success of recommendation. See section 3.1 for more details.

We represent this data set as a directed multi graph. The nodes represent customers, and a directed edge contains all the information about the recommendation. The edge $(i, j, p, t)$ indicates that $i$ recommended product $p$ to customer $j$ at time $t$. Note that as there can be multiple recommendations of between the persons (even on the same product) there can be multiple edges between two nodes.

The typical process generating edges in the recommendation network is as follows: a node $i$ first buys a product $p$ at time $t$ and then it recommends it to nodes $j_{1}, \ldots, j_{n}$. The $j$ nodes can then buy the product and further recommend it. The only way for a node to recommend a product is to first buy it. Note that even if all nodes $j$ buy a product, only the edge to the node $j_{k}$ that first made the purchase (within a week after the recommendation) will be marked by a buy-bit. Because the buy-bit is set only for the first person who acts on a recommendation, we identify additional purchases by the presence of outgoing recommendations for a person, since all recommendations must be preceded by a purchase. We call this type of evidence of purchase a buy-edge. Note that buy-edges provide only a lower bound on the total number of purchases without discounts. It is possible for a customer to not be the first to act on a recommendation and also to not recommend the product to others. Unfortunately, this 


\begin{tabular}{l||rrrrrr}
\hline Group & $p$ & $n$ & $r$ & $e$ & $b_{b}$ & $b_{e}$ \\
\hline Book & 103,161 & $2,863,977$ & $5,741,611$ & $2,097,809$ & 65,344 & 17,769 \\
DVD & 19,829 & 805,285 & $8,180,393$ & 962,341 & 17,232 & 58,189 \\
Music & 393,598 & 794,148 & $1,443,847$ & 585,738 & 7,837 & 2,739 \\
Video & 26,131 & 239,583 & 280,270 & 160,683 & 909 & 467 \\
\hline Full network & 542,719 & $3,943,084$ & $15,646,121$ & $3,153,676$ & 91,322 & 79,164 \\
\hline
\end{tabular}

Table 1: Product group recommendation statistics. $p$ : number of products, $n$ : number of nodes, $r$ : number of recommendations, $e$ : number of edges, $b_{b}$ : number of buy bits, $b_{e}$ : number of buy edges.

was not recorded in the data set. We consider, however, the buy-bits and buy-edges as proxies for the total number of purchases through recommendations.

As mentioned above the first buyer only gets a discount (the buy-bit is turned on) if the purchase is made within one week of the recommendation. In order to account for as many purchases as possible, we consider all purchases where the recommendation preceded the purchase (buy-edge) regardless of the time difference between the two events.

To avoid confusion we will refer to edges in a multi graph as recommendations (or multi-edges) - there can be more than one recommendation between a pair of nodes. We will use the term edge (or unique edge) to refer to edges in the usual sense, i.e. there is only one edge between a pair of people. And, to get from recommendations to edges we create an edge between a pair of people if they exchanged at least one recommendation.

\section{The recommendation network}

For each product group we took recommendations on all products from the group and created a network. Table 1 shows the sizes of various product group recommendation networks with $p$ being the total number of products in the product group, $n$ the total number of nodes spanned by the group recommendation network, and $r$ the number of recommendations (there can be multiple recommendations between two nodes). Column $e$ shows the number of (unique) edges - disregarding multiple recommendations between the same source and recipient (i.e., number of pairs of people that exchanged at least one recommendation).

In terms of the number of different items, there are by far the most music CDs, followed by books and videos. There is a surprisingly small number of DVD titles. On the other hand, DVDs account for more half of all recommendations in the dataset. The DVD network is also the most dense, having about 10 recommendations per node, while books and music have about 2 recommendations per node and videos have only a bit more than 1 recommendation per node.

Music recommendations reached about the same number of people as DVDs but used more than 5 times fewer recommendations to achieve the same coverage of the nodes. Book recommendations reached by far the most people -2.8 million. Notice that all networks have a very small number of unique edges. For books, videos and music the number of unique edges is smaller than the number of nodes - this suggests 


\begin{tabular}{l||rrrrr}
\hline Group & $n_{c}$ & $r_{c}$ & $e_{c}$ & $b_{b c}$ & $b_{e c}$ \\
\hline Book & 53,681 & 933,988 & 184,188 & 1,919 & 1,921 \\
DVD & 39,699 & $6,903,087$ & 442,747 & 6,199 & 41,744 \\
Music & 22,044 & 295,543 & 82,844 & 348 & 456 \\
Video & 4,964 & 23,555 & 15,331 & 2 & 74 \\
\hline Full network & 100,460 & $8,283,753$ & 521,803 & 8,468 & 44,195 \\
\hline
\end{tabular}

Table 2: Statistics for the largest connected component of each product group. $n_{c}$ : number of nodes in largest connected component, $r_{c}$ : number recommendations in the component, $e_{c}$ : number of edges in the component, $b_{b c}$ : number of buy bits, $b_{e c}$ : number of buy edges in the largest connected component, and $b_{b c}$ and $b_{e c}$ are the number of purchase through a buy-bit and a buy-edge, respectively.

that the networks are highly disconnected [ER60.

Back to table 1] given the total number of recommendations $r$ and purchases $\left(b_{b}\right.$ $+b_{e}$ ) influenced by recommendations we can estimate how many recommendations need to be independently sent over the network to induce a new purchase. Using this metric books have the most influential recommendations followed by DVDs and music. For books one out of 69 recommendations resulted in a purchase. For DVDs it increases to 108 recommendations per purchase and further increases to 136 for music and 203 for video.

Table 2 gives more insight into the structure of the largest connected component of each product group's recommendation network. We performed the same measurements as in table 1 with the difference being that we did not use the whole network but only its largest weakly connected component. The table shows the number of nodes $n$, the number of recommendations $r_{c}$, and the number of (unique) edges $e_{c}$ in the largest component. The last two columns $\left(b_{b c}\right.$ and $\left.b_{e c}\right)$ show the number of purchases resulting in a discount (buy-bit, $b_{b c}$ ) and the number of purchases through buy-edges $\left(b_{e c}\right)$ in the largest connected component.

First, notice that the largest connected components are very small. DVDs have the largest - containing $4.9 \%$ of the nodes, books have the smallest at $1.78 \%$. One would also expect that the fraction of the recommendations in the largest component would be proportional to its size. We notice that this is not the case. For example, the largest component in the full recommendation network contains $2.54 \%$ of the nodes and $52.9 \%$ of all recommendations, which is the result of heavy bias in DVD recommendations. Breaking this down by product categories we see that for DVDs $84.3 \%$ of the recommendations are in largest component (which contains $4.9 \%$ of all DVD nodes), vs. $16.3 \%$ for book recommendations (component size $1.79 \%$ ), $20.5 \%$ for music recommendations (component size $2.77 \%$ ), and $8.4 \%$ for video recommendations (component size $2.1 \%$ ). This shows that the dynamic in the largest component is very much different from the rest of the network. Especially for DVDs we can see that a very small fraction of users generated most of the recommendations.

\subsection{Recommendation network over time}

The recommendations that occurred were exchanged over an existing underlying social network. In the real world, it is estimated that any two people on the globe 


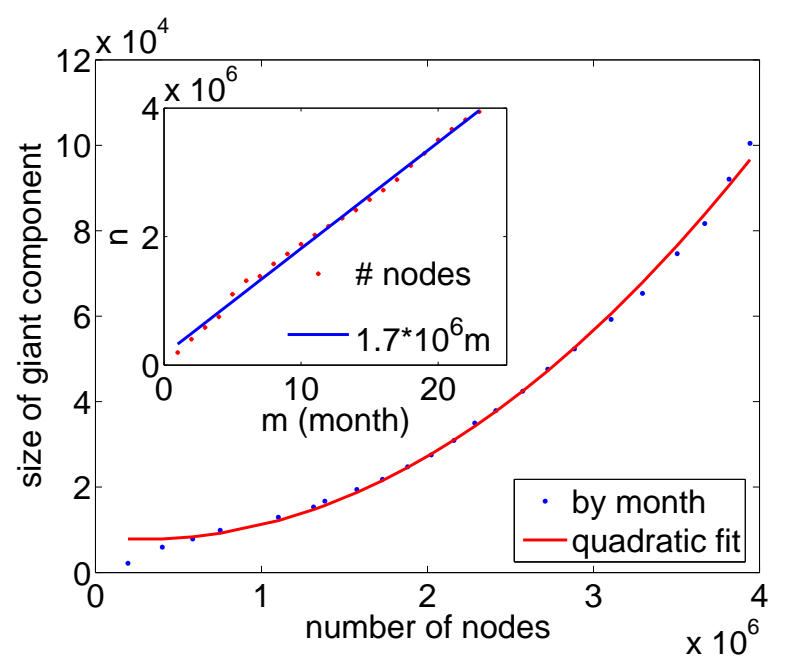

Figure 1: (a) The size of the largest connected component of customers over time. The inset shows the linear growth in the number of customers $n$ over time.

are connected via a short chain of acquaintances - popularly known as the small world phenomenon TM69. We examined whether the edges formed by aggregating recommendations over all products would similarly yield a small world network, even though they represent only a small fraction of a person's complete social network. We measured the growth of the largest weakly connected component over time, shown in Figure 1. Within the weakly connected component, any node can be reached from any other node by traversing (undirected) edges. For example, if $u$ recommended product $x$ to $v$, and $w$ recommended product $y$ to $v$, then $u$ and $w$ are linked through one intermediary and thus belong to the same weakly connected component. Note that connected components do not necessarily correspond to communities (clusters) which we often think of as densely linked parts of the networks. Nodes belong to same component if they can reach each other via an undirected path regardless of how densely they are linked.

Figure 1 shows the size of the largest connected component, as a fraction of the total network. The largest component is very small over all time. Even though we compose the network using all the recommendations in the dataset, the largest connected component contains less than $2.5 \%(100,420)$ of the nodes, and the second largest component has only 600 nodes. Still, some smaller communities, numbering in the tens of thousands of purchasers of DVDs in categories such as westerns, classics and Japanese animated films (anime), had connected components spanning about $20 \%$ of their members.

The insert in figure 1 shows the growth of the customer base over time. Surprisingly it was linear, adding on average 165,000 new users each month, which is an indication that the service itself was not spreading epidemically. Further evidence of non-viral spread is provided by the relatively high percentage (94\%) of users who made their first recommendation without having previously received one. 


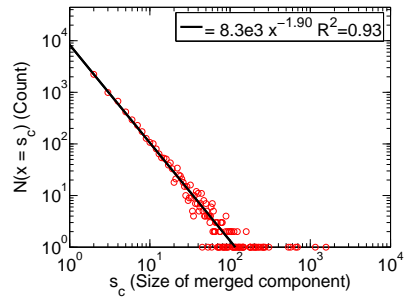

(a) LCC growth

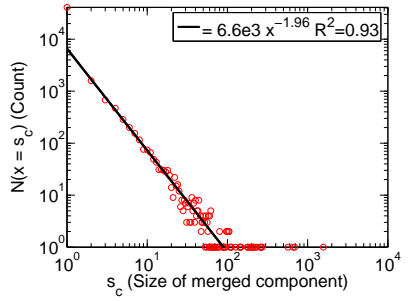

(b) Sender in LCC

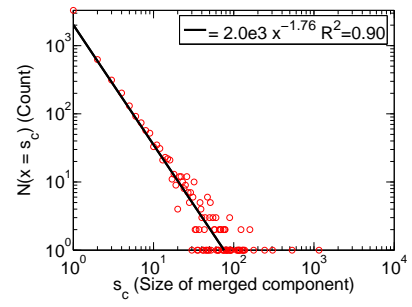

(c) Sender outside LCC

Figure 2: Growth of the largest connected component (LCC). (a) the distribution of sizes of components when they are merged into the largest connected component. (b) same as (a), but restricted to cases when a member of the LCC sends a recommendation to someone outside the largest component. (c) a sender outside the largest component sends a recommendation to a member of the component.

\subsubsection{Growth of the largest connected component}

Next, we examine the growth of the largest connected component (LCC). In figure 1 we saw that the largest component seems to grow quadratically over time, but at the end of the data collection period is still very small, i.e. only $2.5 \%$ of the nodes belong to largest weakly connected component. Here we are not interested in how fast the largest component grows over time but rather how big other components are when they get merged into the largest component. Also, since our graph is directed we are interested in determining whether smaller components become attached to the largest component by a recommendation sent from inside of the largest component. One can think of these recommendations as being tentacles reaching out of largest component to attach smaller components. The other possibility is that the recommendation comes from a node outside the component to a member of the largest component and thus the initiative to attach comes from outside the largest component.

We look at whether the largest component grows gradually, adding nodes one by one as the members send out more recommendations, or whether a new recommendation might act as a bridge to a component consisting of several nodes who are already

linked by their previous recommendations. To this end we measure the distribution of a component's size when it gets merged to the largest weakly connected component.

We operate under the following setting. Recommendations are arriving over time one by one creating edges between the nodes of the network. As more edges are being added the size of largest connected component grows. We keep track of the currently largest component, and measure how big the separate components are when they get attached to the largest component.

Figure 2(a) shows the distribution of merged connected component (CC) sizes. On the $\mathrm{x}$-axis we plot the component size (number of nodes $N$ ) and on the y-axis the number of components of size $N$ that were merged over time with the largest component. We see that a majority of the time a single node (component of size 1) merged with the currently largest component. On the other extreme is the case when a component of 1,568 nodes merged with the largest component.

Interestingly, out of all merged components, in $77 \%$ of the cases the source of the 
recommendation comes from inside the largest component, while in the remaining $23 \%$ of the cases it is the smaller component that attaches itself to the largest one. Figure 2(b) shows the distribution of component sizes only for the case when the sender of the recommendation was a member of the largest component, i.e. the small component was attached from the largest component. Lastly, Figure 2(c) shows the distribution for the opposite case when the sender of the recommendation was not a member of the largest component, i.e. the small component attached itself to the largest.

Also notice that in all cases the distribution of merged component sizes follows a heavy-tailed distribution. We fit a power-law distribution and note the power-law exponent of 1.90 (fig. 2(a)) when considering all merged components. Limiting the analysis to the cases where the source of the edge that attached a small component to the largest is in the largest component we obtain power-law exponent of 1.96 (fig. 2(b)), and when the edge originated from the small component to attached it to the largest, the power-law exponent is 1.76. This shows that even though in most cases the LCC absorbs the small component, we see that components that attach themselves to the LCC tend to be larger (smaller power-law exponent) than those attracted by the LCC. This means that the component sometimes grows a bit before it attaches itself to the largest component. Intuitively, an individual node can get attached to the largest component simply by passively receiving a recommendation. But if it is the outside node that sends a recommendation to someone in the giant component, it is already an active recommender and could therefore have recommended to several others previously, thus forming a slightly bigger component that is then merged.

From these experiments we see that the largest component is very active, adding smaller components by generating new recommendations. Most of the time these newly merged components are quite small, but occasionally sizable components are attached.

\subsection{Preliminary observations and discussion}

Even with these simple counts and experiments we can already make a few observations. It seems that some people got quite heavily involved in the recommendation program, and that they tended to recommend a large number of products to the same set of friends (since the number of unique edges is so small as shown on table1). This means that people tend to buy more DVDs and also like to recommend them to their friends, while they seem to be more conservative with books. One possible reason is that a book is a bigger time investment than a DVD: one usually needs several days to read a book, while a DVD can be viewed in a single evening. Another factor may be how informed the customer is about the product. DVDs, while fewer in number, are more heavily advertised on TV, billboards, and movie theater previews. Furthermore, it is possible that a customer has already watched a movie and is adding the DVD to their collection. This could make them more confident in sending recommendations before viewing the purchased DVD.

One external factor which may be affecting the recommendation patterns for DVDs is the existence of referral websites (www.dvdtalk.com). On these websites people, who want to buy a DVD and get a discount, would ask for recommendations. This way there would be recommendations made between people who don't really know 


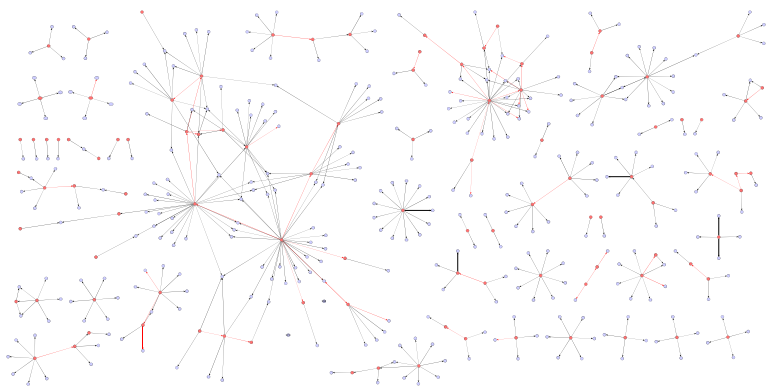

(a) Medical book

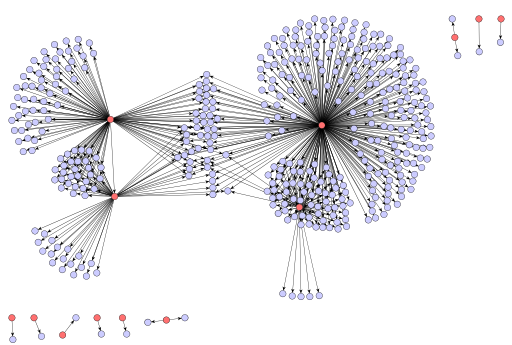

(b) Japanese graphic novel

Figure 3: Examples of two product recommendation networks: (a) First aid study guide First Aid for the USMLE Step, (b) Japanese graphic novel (manga) Oh My Goddess!: Mara Strikes Back.

\begin{tabular}{l|rrr}
\hline & \multicolumn{3}{|c}{ Number of nodes } \\
Group & Purchases & Forward & Percent \\
\hline Book & 65,391 & 15,769 & 24.2 \\
DVD & 16,459 & 7,336 & 44.6 \\
Music & 7,843 & 1,824 & 23.3 \\
Video & 909 & 250 & 27.6 \\
\hline Total & 90,602 & 25,179 & 27.8 \\
\hline
\end{tabular}

Table 3: Fraction of people that purchase and also recommend forward. Purchases: number of nodes that purchased as a result of receiving a recommendation. Forward: nodes that purchased and then also recommended the product to others.

each other but rather have an economic incentive to cooperate.

In effect, the viral marketing program is altering, albeit briefly and most likely unintentionally, the structure of the social network it is spreading on. We were not able to find similar referral sharing sites for books or CDs.

\section{Propagation of recommendations}

\subsection{Forward recommendations}

Not all people who accept a recommendation by making a purchase also decide to give recommendations. In estimating what fraction of people that purchase also decide to recommend forward, we can only use the nodes with purchases that resulted in a discount. Table 3 shows that only about a third of the people that purchase also recommend the product forward. The ratio of forward recommendations is much higher for DVDs than for other kinds of products. Videos also have a higher ratio of forward recommendations, while books have the lowest. This shows that people are most keen on recommending movies, possibly for the above mentioned reasons, while more conservative when recommending books and music.

Figure 4 shows the cumulative out-degree distribution, that is the number of 


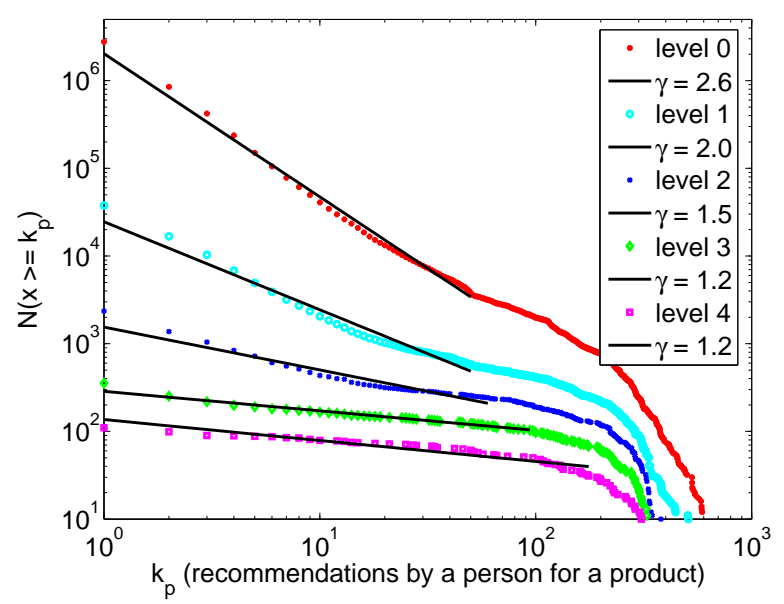

Figure 4: The number of recommendations sent by a user with each curve representing a different depth of the user in the recommendation chain. A power law exponent $\gamma$ is fitted to all but the tail, which shows an exponential drop-off at around 100 recommendations sent). This drop-off is consistent across all depth levels, and may reflect either a natural disinclination to send recommendation to over a hundred people, or a technical issue that might have made it more inconvenient to do so. The fitted lines follow the order of the level number (i.e. top line corresponds to level 0 and bottom to level 4).

\begin{tabular}{c|rr}
\hline level & $\begin{array}{r}\text { prob. buy \& } \\
\text { forward }\end{array}$ & $\begin{array}{r}\text { average } \\
\text { out-degree }\end{array}$ \\
\hline 0 & $\mathrm{~N} / \mathrm{A}$ & 1.99 \\
1 & 0.0069 & 5.34 \\
2 & 0.0149 & 24.43 \\
3 & 0.0115 & 72.79 \\
4 & 0.0082 & 111.75
\end{tabular}

Table 4: Statistics about individuals at different levels of the cascade.

people who sent out at least $k_{p}$ recommendations, for a product. We fit a power-law to all but the tail of the distribution. Also, notice the exponential decay in the tail of the distribution which could be, among other reasons, attributed to the finite time horizon of our dataset.

The figure 4 shows that the deeper an individual is in the cascade, if they choose to make recommendations, they tend to recommend to a greater number of people on average (the fitted line has smaller slope $\gamma$, i.e. the distribution has higher variance). This effect is probably due to only very heavily recommended products producing large enough cascades to reach a certain depth. We also observe, as is shown in Table 4, that the probability of an individual making a recommendation at all (which can only occur if they make a purchase), declines after an initial increase as one gets deeper into the cascade. 


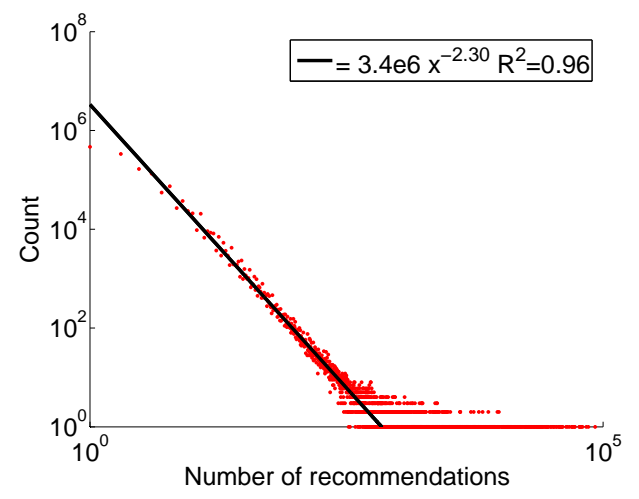

(a) Recommendations

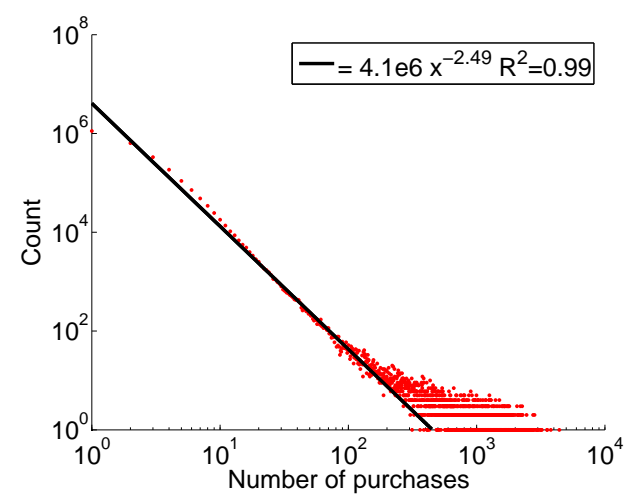

(b) Purchases

Figure 5: Distribution of the number of recommendations and number of purchases made by a customer.

\subsection{Identifying cascades}

As customers continue forwarding recommendations, they contribute to the formation of cascades. In order to identify cascades, i.e. the "causal" propagation of recommendations, we track successful recommendations as they influence purchases and further recommendations. We define a recommendation to be successful if it reached a node before its first purchase. We consider only the first purchase of an item, because there are many cases when a person made multiple purchases of the same product, and in between those purchases she may have received new recommendations. In this case one cannot conclude that recommendations following the first purchase influenced the later purchases.

Each cascade is a network consisting of customers (nodes) who purchased the same product as a result of each other's recommendations (edges). We delete late recommendations - all incoming recommendations that happened after the first purchase of the product. This way we make the network time increasing or causal - for each node all incoming edges (recommendations) occurred before all outgoing edges. Now each connected component represents a time obeying propagation of recommendations.

Figure 3 shows two typical product recommendation networks: (a) a medical study guide and (b) a Japanese graphic novel. Throughout the dataset we observe very similar patters. Most product recommendation networks consist of a large number of small disconnected components where we do not observe cascades. Then there is usually a small number of relatively small components with recommendations successfully propagating. This observation is reflected in the heavy tailed distribution of cascade sizes (see figure 6), having a power-law exponent close to 1 for DVDs in particular. We determined the power-law exponent by fitting a line on log-log scales using the least squares method.

We also notice bursts of recommendations (figure 3(b)). Some nodes recommend to many friends, forming a star like pattern. Figure 5 shows the distribution of 


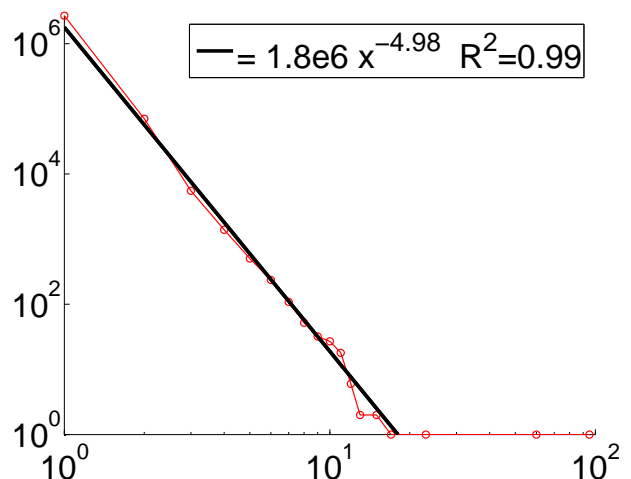

(a) Book

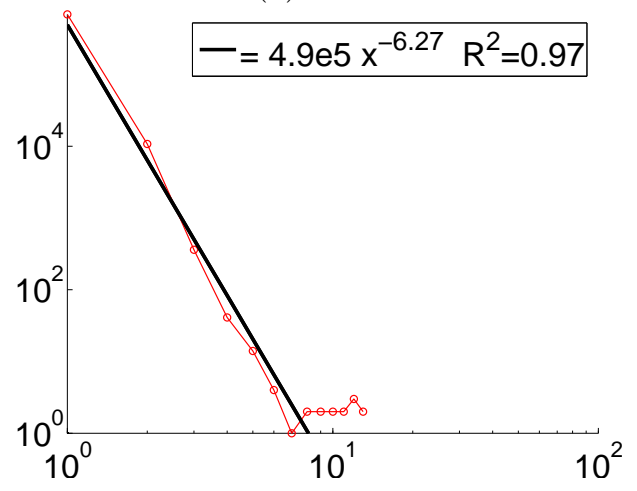

(c) Music

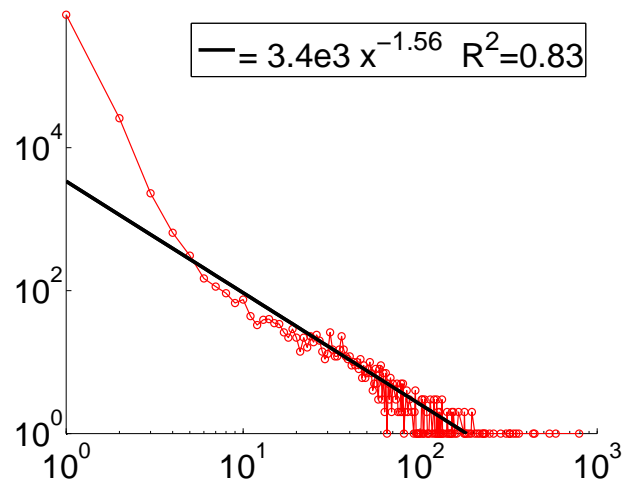

(b) DVD

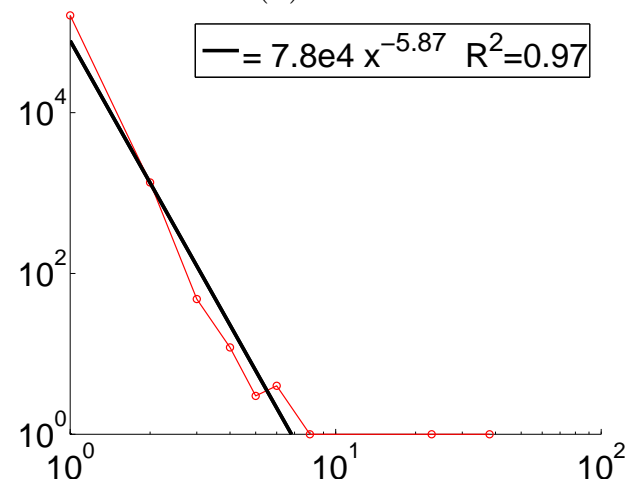

(d) Video

Figure 6: Size distribution of cascades (size of cascade vs. count). Bold line presents a power-fit.

the recommendations and purchases made by a single node in the recommendation network. Notice the power-law distributions and long flat tails. The most active customer made 83,729 recommendations and purchased 4,416 different items. Finally, we also sometimes observe 'collisions', where nodes receive recommendations from two or more sources. A detailed enumeration and analysis of observed topological cascade patterns for this dataset is made in LSK06.

Last, we examine the number of exchanged recommendations between a pair of people in figure 7. Overall, $39 \%$ of pairs of people exchanged just a single recommendation. This number decreases for DVDs to $37 \%$, and increases for books to $45 \%$. The distribution of the number of exchanged recommendations follows a heavy tailed distribution. To get a better understanding of the distributions we show the power-law decay lines. Notice that one gets much stronger decay exponent (distribution has weaker tail) of -2.7 for books and a very shallow power-law exponent of -1.5 for DVDs. This means that even a pair of people exchanges more DVD than book recommendations. 


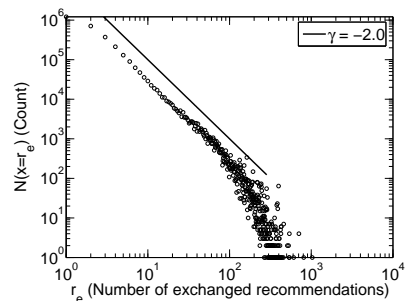

(a) All

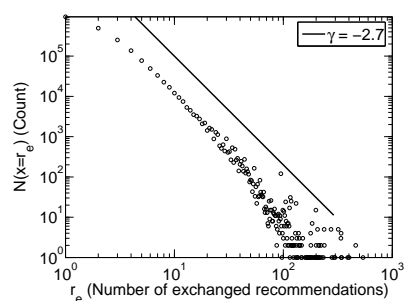

(b) Books

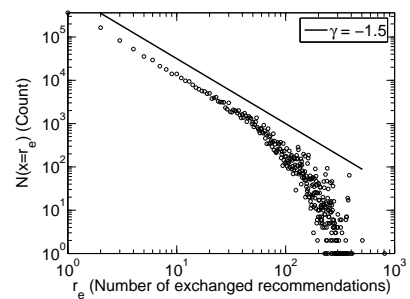

(c) DVD

Figure 7: Distribution of the number of exchanged recommendations between pairs of people.

\subsection{The recommendation propagation model}

A simple model can help explain how the wide variance we observe in the number of recommendations made by individuals can lead to power-laws in cascade sizes (figure 6). The model assumes that each recipient of a recommendation will forward it to others if its value exceeds an arbitrary threshold that the individual sets for herself. Since exceeding this value is a probabilistic event, let's call $p_{t}$ the probability that at time step $t$ the recommendation exceeds the threshold. In that case the number of recommendations $N_{t+1}$ at time $(t+1)$ is given in terms of the number of recommendations at an earlier time by

$$
N_{t+1}=p_{t} N_{t}
$$

where the probability $p_{t}$ is defined over the unit interval.

Notice that, because of the probabilistic nature of the threshold being exceeded, one can only compute the final distribution of recommendation chain lengths, which we now proceed to do.

Subtracting from both sides of this equation the term $N_{t}$ and diving by it we obtain

$$
\frac{N_{(t+1)}-N_{t}}{N_{t}}=p_{t}-1
$$

Summing both sides from the initial time to some very large time $T$ and assuming that for long times the numerator is smaller than the denominator (a reasonable assumption) we get, up to a unit constant

$$
\frac{d N}{N}=\sum p_{t}
$$

The left hand integral is just $\log (N)$, and the right hand side is a sum of random variables, which in the limit of a very large uncorrelated number of recommendations is normally distributed (central limit theorem).

This means that the logarithm of the number of messages is normally distributed, or equivalently, that the number of messages passed is log-normally distributed. In other words the probability density for $N$ is given by 


$$
P(N)=\frac{1}{N \sqrt{2 \pi \sigma^{2}}} \exp \frac{-(\log (N)-\mu)^{2}}{2 \sigma^{2}}
$$

which, for large variances describes a behavior whereby the typical number of recommendations is small (the mode of the distribution) but there are unlikely events of large chains of recommendations which are also observable.

Furthermore, for large variances, the lognormal distribution can behave like a power law for a range of values. In order to see this, take the logarithms on both sides of the equation (equivalent to a log-log plot) and one obtains

$$
\log (P(N))=-\log (N)-\log \left(\sqrt{2 \pi \sigma^{2}}\right)-\frac{(\log (N)-\mu)^{2}}{2 \sigma^{2}}
$$

So, for large $\sigma$, the last term of the right hand side goes to zero, and since the second term is a constant one obtains a power law behavior with exponent value of minus one. There are other models which produce power-law distributions of cascade sizes, but we present ours for its simplicity, since it does not depend on network topology GGLNT04 or critical thresholds in the probability of a recommendation being accepted Wat02].

\section{Success of Recommendations}

So far we only looked into the aggregate statistics of the recommendation network. Next, we ask questions about the effectiveness of recommendations in the recommendation network itself. First, we analyze the probability of purchasing as one gets more and more recommendations. Next, we measure recommendation effectiveness as two people exchange more and more recommendations. Lastly, we observe the recommendation network from the perspective of the sender of the recommendation. Does a node that makes more recommendations also influence more purchases?

\subsection{Probability of buying versus number of incoming recom- mendations}

First, we examine how the probability of purchasing changes as one gets more and more recommendations. One would expect that a person is more likely to buy a product if she gets more recommendations. On the other had one would also think that there is a saturation point - if a person hasn't bought a product after a number of recommendations, they are not likely to change their minds after receiving even more of them. So, how many recommendations are too many?

Figure 8 shows the probability of purchasing a product as a function of the number of incoming recommendations on the product. Because we exclude late recommendations, those that were received after the purchase, an individual counts as having received three recommendations only if they did not make a purchase after the first two, and either purchased or did not receive further recommendations after receiving the third one. As we move to higher numbers of incoming recommendations, the number of observations drops rapidly. For example, there were 5 million cases with 1 incoming recommendation on a book, and only 58 cases where a person got 20 


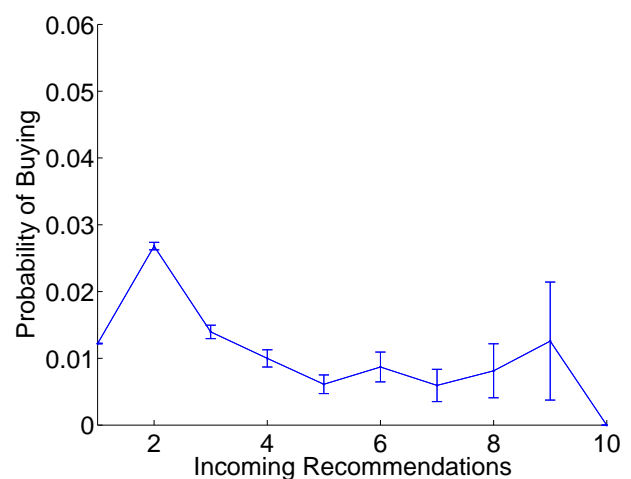

(a) Books

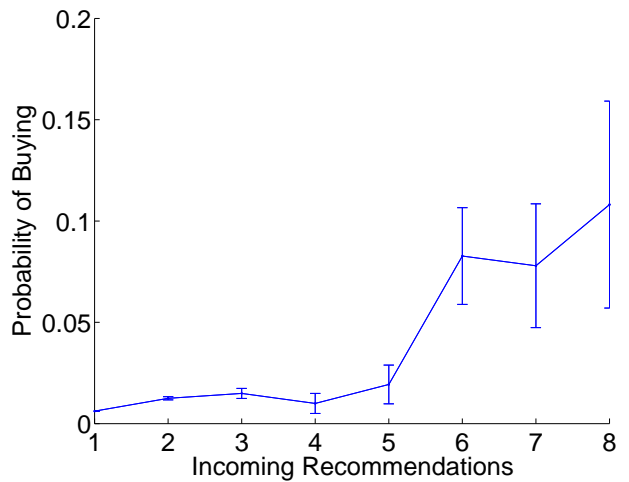

(c) Music

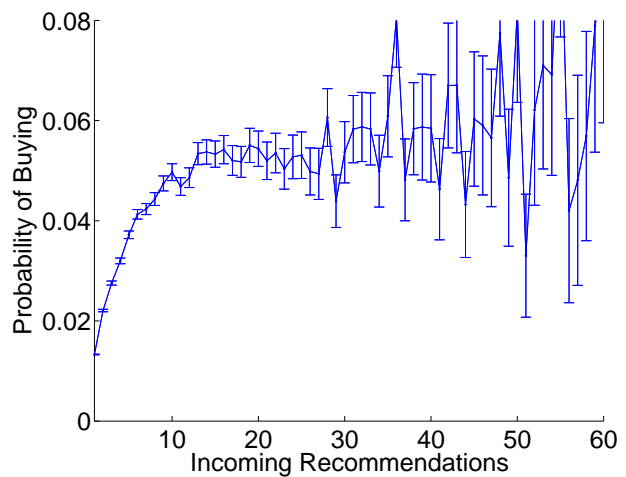

(b) DVD

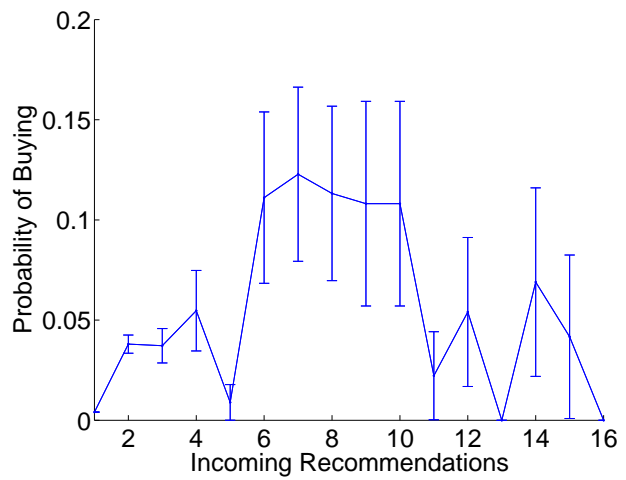

(d) Video

Figure 8: Probability of buying a book (DVD) given a number of incoming recommendations.

incoming recommendations on a particular book. The maximum was 30 incoming recommendations. For these reasons we cut-off the plot when the number of observations becomes too small and the error bars too large.

We calculate the purchase probabilities and the standard errors of the estimates which we use to plot the error bars in the following way. We regard each point as a binomial random variable. Given the number of observations $n$, let $m$ be the number of successes, and $\mathrm{k}(\mathrm{k}=\mathrm{n}-\mathrm{m})$ the number of failures. In our case, $\mathrm{m}$ is the number of people that first purchased a product after receiving $\mathrm{r}$ recommendations on it, and $\mathrm{k}$ is the number of people that received the total of $r$ recommendations on a product (till the end of the dataset) but did purchase it, then the estimated probability of purchasing is $\hat{p}=m / n$ and the standard error $s_{\hat{p}}$ of estimate $\hat{p}$ is $s_{\hat{p}}=\sqrt{p(1-p) / n}$.

Figure 8(a) shows that, overall, book recommendations are rarely followed. Even more surprisingly, as more and more recommendations are received, their success decreases. We observe a peak in probability of buying at 2 incoming recommendations and then a slow drop. This implies that if a person doesn't buy a book after the first recommendation, but receives another, they are more likely to be persuaded by the second recommendation. But thereafter, they are less likely to respond to additional 
recommendations, possibly because they perceive them as spam, are less susceptible to others' opinions, have a strong opinion on the particular product, or have a different means of accessing it.

For DVDs (figure 8(b)) we observe a saturation around 10 incoming recommendations. This means that with each additional recommendation, a person is more and more likely to be persuaded - up to a point. After a person gets 10 recommendations on a particular DVD, their probability of buying does not increase anymore. The number of observations is 2.5 million at 1 incoming recommendation and 100 at 60 incoming recommendations. The maximal number of received recommendations is 172 (and that person did not buy), but someone purchased a DVD after 169 receiving recommendations. The different patterns between book and DVD recommendations may be a result of the recommendation exchange websites for DVDs. Someone receiving many DVD recommendations may have signed up to receive them for a product they intended to purchase, and hence a greater number of received recommendations corresponds to a higher likelihood of purchase (up to a point).

\subsection{Success of subsequent recommendations}

Next, we analyze how the effectiveness of recommendations changes as one received more and more recommendations from the same person. A large number of exchanged recommendations can be a sign of trust and influence, but a sender of too many recommendations can be perceived as a spammer. A person who recommends only a few products will have her friends' attention, but one who floods her friends with all sorts of recommendations will start to loose her influence.

We measure the effectiveness of recommendations as a function of the total number of previously received recommendations from a particular node. We thus measure how spending changes over time, where time is measured in the number of received recommendations.

We construct the experiment in the following way. For every recommendation $r$ on some product $p$ between nodes $u$ and $v$, we first determine how many recommendations node $u$ received from $v$ before getting $r$. Then we check whether $v$, the recipient of recommendation, purchased $p$ after the recommendation $r$ arrived. If so, we count the recommendation as successful since it influenced the purchase. This way we can calculate the recommendation success rate as more recommendations were exchanged. For the experiment we consider only node pairs $(u, v)$, where there were at least a total of 10 recommendations sent from $u$ to $v$. We perform the experiment using only recommendations from the same product group.

We decided to set a lower limit on the number of exchanged recommendations so that we can measure how the effectiveness of recommendations changes as the same two people exchange more and more recommendations. Considering all pairs of people would heavily bias our findings since most pairs exchange just a few or even just a single recommendation. Using the data from figure 7 we see that $91 \%$ of pairs of people that exchange at least 1 recommendation exchange less than 10. For books this number increases to $96 \%$, and for DVDs it is even smaller (81\%). In the DVD network there are 182 thousand pairs that exchanged more than 10 recommendations, and 70 thousand for the book network.

Figure 9 shows the probability of buying as a function of the total number of 


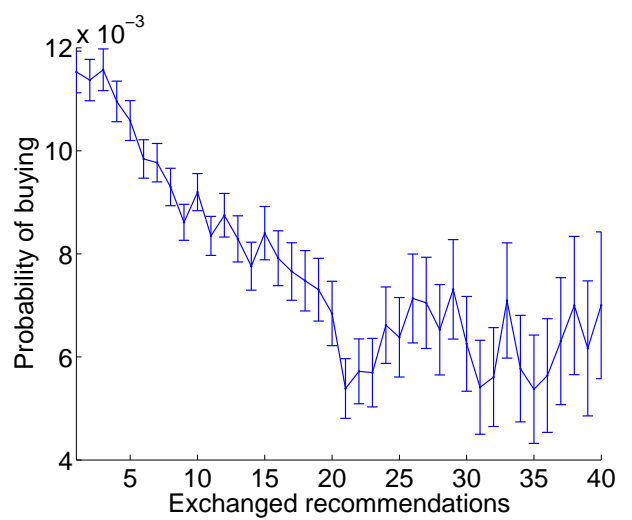

(a) Books

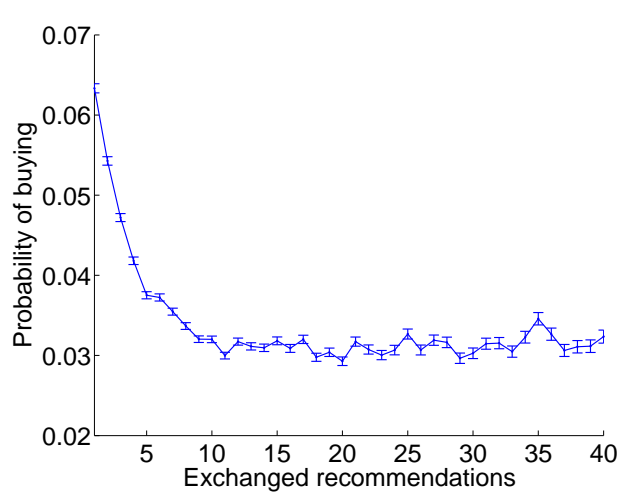

(b) DVD

Figure 9: The effectiveness of recommendations with the number of received recommendations.

received recommendations from a particular person up to that point. One can think of $\mathrm{x}$-axis as measuring time where the unit is the number of received recommendations from a particular person.

For books we observe that the effectiveness of recommendation remains about constant up to 3 exchanged recommendations. As the number of exchanged recommendations increases, the probability of buying starts to decrease to about half of the original value and then levels off. For DVDs we observe an immediate and consistent drop. We performed the experiment also for video and music, but the number of observations was too low and the measurements were noisy. This experiment shows that recommendations start to lose effect after more than two or three are passed between two people. Also, notice that the effectiveness of book recommendations decays much more slowly than that of DVD recommendations, flattening out at around 20 recommendations, compared to around 10 DVD exchanged recommendations.

The result has important implications for viral marketing because providing too much incentive for people to recommend to one another can weaken the very social network links that the marketer is intending to exploit.

\subsection{Success of outgoing recommendations}

In previous sections we examined the data from the viewpoint of the receiver of the recommendation. Now we look from the viewpoint of the sender. The two interesting questions are: how does the probability of getting a $10 \%$ credit change with the number of outgoing recommendations; and given a number of outgoing recommendations, how many purchases will they influence?

One would expect that recommendations would be the most effective when recommended to the right subset of friends. If one is very selective and recommends to too few friends, then the chances of success are slim. One the other hand, recommending to everyone and spamming them with recommendations may have limited returns as well. 

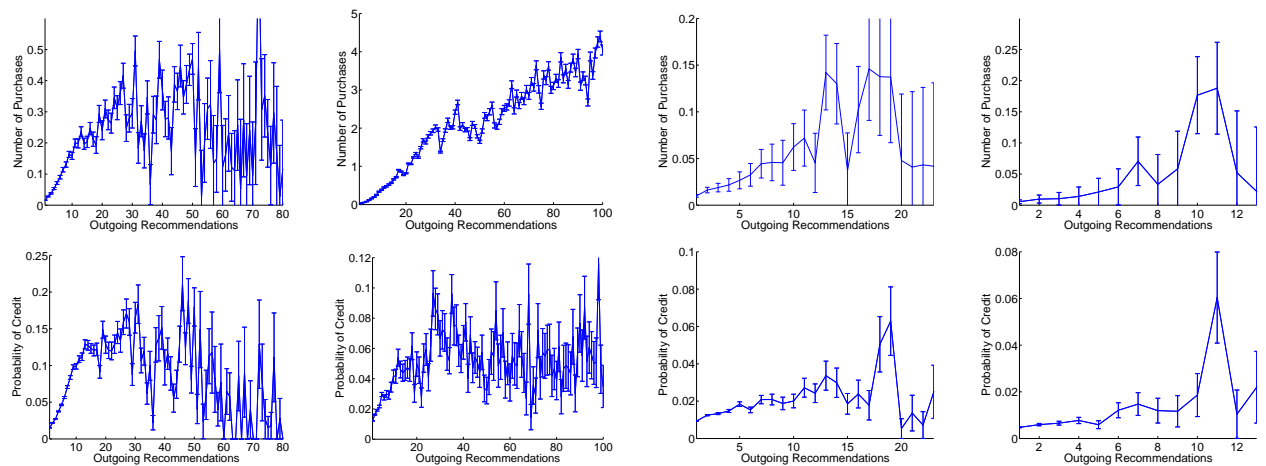

(a) Books

(b) DVD

(c) Music

(d) Video

Figure 10: Top row: Number of resulting purchases given a number of outgoing recommendations. Bottom row: Probability of getting a credit given a number of outgoing recommendations.

The top row of figure 10 shows how the average number of purchases changes with the number of outgoing recommendations. For books, music, and videos the number of purchases soon saturates: it grows fast up to around 10 outgoing recommendations and then the trend either slows or starts to drop. DVDs exhibit different behavior, with the expected number of purchases increasing throughout.

These results are even more interesting since the receiver of the recommendation does not know how many other people also received the recommendation. Thus the plots of figure 10 show that there are interesting dependencies between the product characteristics and the recommender that manifest through the number of recommendations sent. It could be the case that widely recommended products are not suitable for viral marketing (we find something similar in section 9.2), or that the recommender did not put too much thought into who to send the recommendation to, or simply that people soon start to ignore mass recommenders.

Plotting the probability of getting a $10 \%$ credit as a function of the number of outgoing recommendations, as in the bottom row of figure 10, we see that the success of DVD recommendations saturates as well, while books, videos and music have qualitatively similar trends. The difference in the curves for DVD recommendations points to the presence of collisions in the dense DVD network, which has 10 recommendations per node and around 400 per product — an order of magnitude more than other product groups. This means that many different individuals are recommending to the same person, and after that person makes a purchase, even though all of them made a 'successful recommendation' by our definition, only one of them receives a credit.

\subsection{Probability of buying given the total number of incoming recommendations}

The collisions of recommendations are a dominant feature of the DVD recommendation network. Book recommendations have the highest chance of getting a credit, but DVD recommendations cause the most purchases. So far it seems people are 


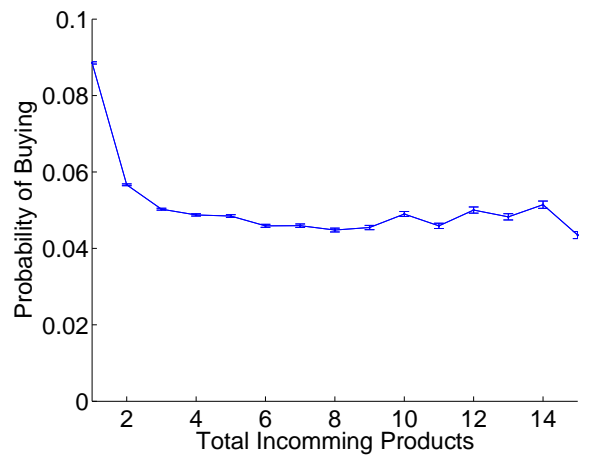

(a) Books

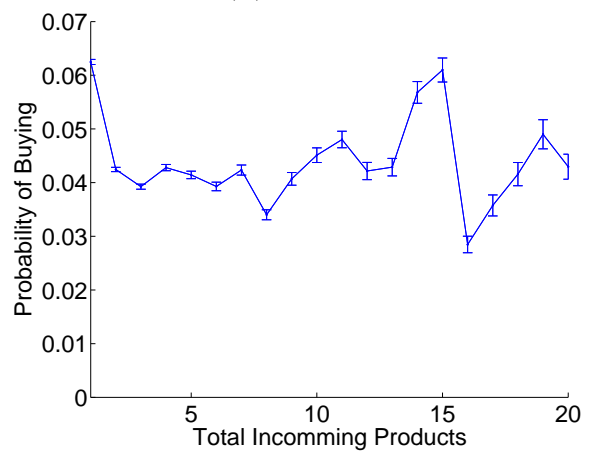

(c) Music

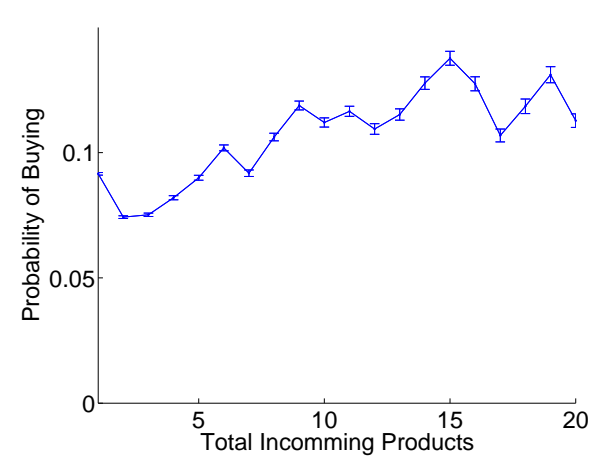

(b) DVD

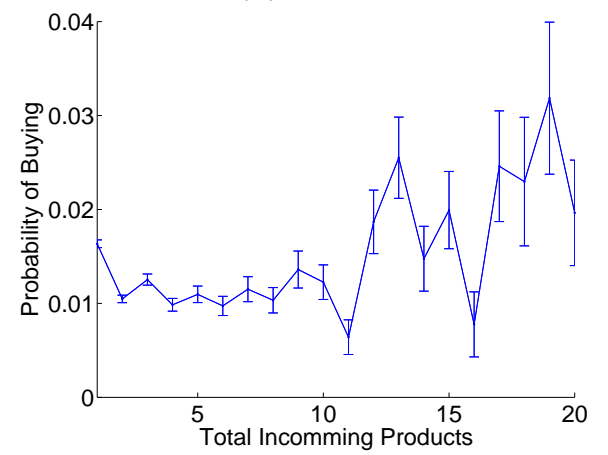

(d) Video

Figure 11: The probability of buying a product given a number of different products a node got recommendations on.

very keen on recommending various DVDs, while very conservative on recommending books. But how does the behavior of customers change as they get more involved into the recommendation network? We would expect that most of the people are not heavily involved, so their probability of buying is not high. In the extreme case we expect to find people who buy almost everything they get recommendations on.

There are two ways to measure the involvedness of a person in the network: by the total number of incoming recommendations (on all products) or the total number of different products they were recommended. For every purchase of a book at time $t$, we count the number of different books (DVDs, ...) the person received recommendations for before time $t$. As in all previous experiments we delete late recommendations, i.e. recommendations that arrived after the first purchase of a product.

We show the probability of buying as a function of the number of different products recommended in Figure 11, Figure A-2 plots the same data but with the total number of incoming recommendations on the x-axis. We calculate the error bars as described in section 6.1. The number of observations is large enough (error bars are sufficiently small) to draw conclusions about the trends observed in the figures. For example, there are more than 15, 000 observations (users) that had 15 incoming DVD recommendations. 


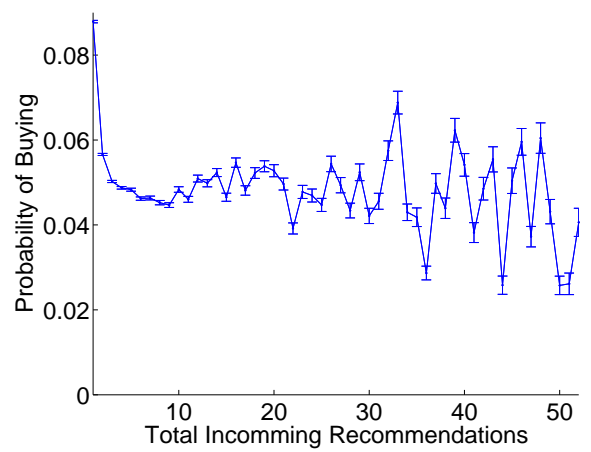

(a) Books

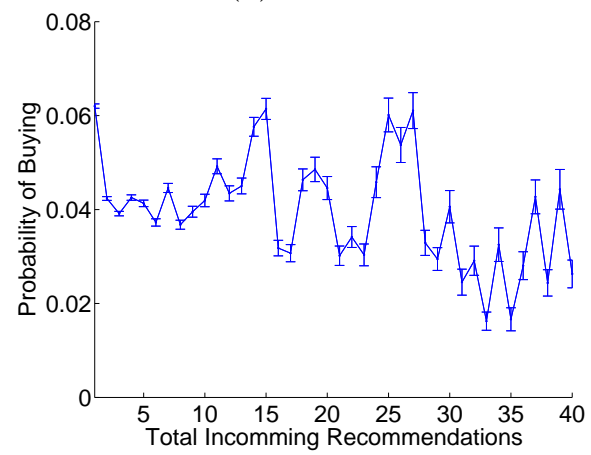

(c) Music

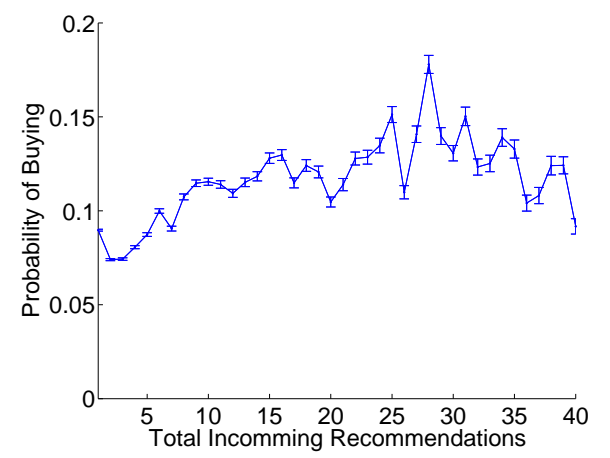

(b) DVD

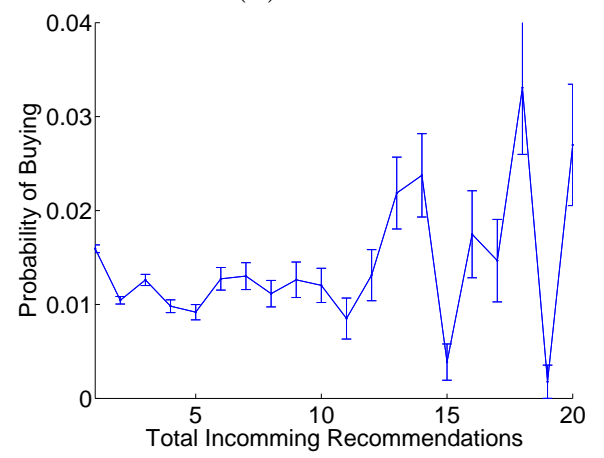

(d) Video

Figure 12: Probability of buying a product given a total number of incoming recommendations on all products.

Notice that trends are quite similar regardless whether we measure how involved is the user in the network by counting the number of products recommended (figure 11) or the number of incoming recommendations (fig. A-2).

We observe two distinct trends. For books and music (figures [1] and A-2, (a) and (c)) the probability of buying is the highest when a person got recommendations on just 1 item, as the number of incoming recommended products increases to 2 or more the probability of buying quickly decreases and then flattens.

Movies (DVDs and videos) exhibit different behavior (figure 11 and A-2, (b) and (d)). A person is more likely to buy the more recommendations she gets. For DVDs the peak is at around 15 incoming products, while for videos there is no such peak the probability remains fairly level. Interestingly for DVDs the distribution reaches its low at 2 and 3 items, while for videos it lies somewhere between 3 and 8 items. The results suggest that books and music buyers tend to be conservative and focused. On the other hand there are people who like to buy movies in general. One could hypothesize that buying a book is a larger investment of time and effort than buying a movie. One can finish a movie in an evening, while reading a book requires more effort. There are also many more book and music titles than movie titles.

The other difference between the book and music recommendations in compar- 
ison to movies are the recommendation referral websites where people could go to get recommendations. One could see these websites as recommendation subscription services - posting one's email on a list results in a higher number of incoming recommendations. For movies, people with a high number of incoming recommendations "subscribed" to them and thus expected/wanted the recommendations. On the other hand people with high numbers of incoming book or music recommendations did not "sign up" for them, so they may perceive recommendations as spam and thus the influence of recommendations drops.

Another evidence of the existence of recommendations referral websites includes the DVD recommendation network degree distribution. The DVDs follow a power law degree distribution with an exception of a peak at out-degree 50. Other plots of DVD recommendation behavior also exhibited abnormalities at around 50 recommendations. We believe these can be attributed to the recommendation referral websites.

\section{Timing of recommendations and purchases}

The recommendation referral program encourages people to purchase as soon as possible after they get a recommendation, since this maximizes the probability of getting a discount. We study the time lag between the recommendation and the purchase of different product groups, effectively how long it takes a person to receive a recommendation, consider it, and act on it.

We present the histograms of the "thinking time", i.e. the difference between the time of purchase and the time the last recommendation was received for the product prior to the purchase (figure 13). We use a bin size of 1 day. Around $35 \%-40 \%$ of book and DVD purchases occurred within a day after the last recommendation was received. For DVDs $16 \%$ purchases occur more than a week after the last recommendation, while this drops to $10 \%$ for books. In contrast, if we consider the lag between the purchase and the first recommendation, only $23 \%$ of DVD purchases are made within a day, while the proportion stays the same for books. This reflects a greater likelihood for a person to receive multiple recommendations for a DVD than for a book. At the same time, DVD recommenders tend to send out many more recommendations, only one of which can result in a discount. Individuals then often miss their chance of a discount, which is reflected in the high ratio (78\%) of recommended DVD purchases that did not a get discount (see table 1 columns $b_{b}$ and $b_{e}$ ). In contrast, for books, only $21 \%$ of purchases through recommendations did not receive a discount.

We also measure the variation in intensity by time of day for three different activities in the recommendation system: recommendations (figure 14(a)), all purchases (figure 14(b)), and finally just the purchases which resulted in a discount (figure 14(c)). Each is given as a total count by hour of day.

The recommendations and purchases follow the same pattern. The only small difference is that purchases reach a sharper peak in the afternoon (after 3pm Pacific Time, $6 \mathrm{pm}$ Eastern time). This means that the willingness to recommend does not change with time, since about a constant fraction of purchases also result in recommendations sent (plots 14(a) and (b) follow the same shape).

The purchases that resulted in a discount (fig. 14(c)) look like a negative image of the first two figures. If recommendations would have no effect then plot (c) should follow the same shape as (a) and (b), since a fraction of people that buy would 


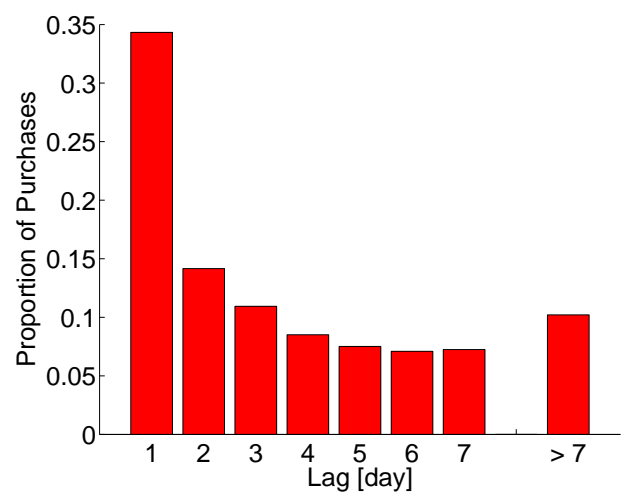

(a) Books

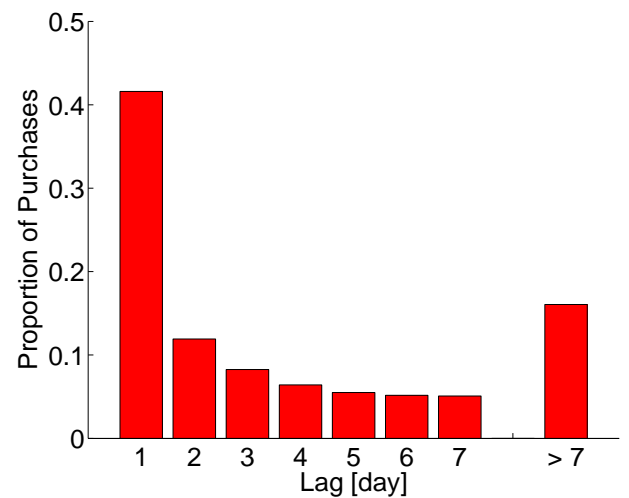

(b) DVD

Figure 13: The time between the recommendation and the actual purchase. We use all purchases.

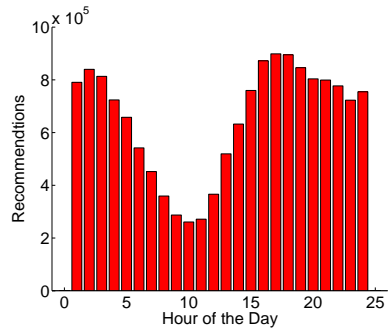

(a) Recommendations

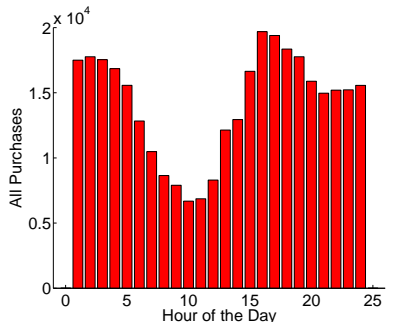

(b) Purchases

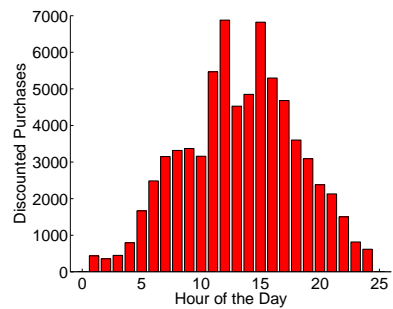

(c) Purchases with Discount

Figure 14: Time of day for purchases and recommendations. (a) shows the distribution of recommendations over the day, (b) shows all purchases and (c) shows only purchases that resulted in a discount.

become first buyers, i.e. the more recommendations sent, the more first buyers and thus discounts. However, this does not seem to be the case. The number of purchases with discount is the high when the number of purchases is small. This means that most of discounted purchases happened in the morning when the traffic (number of purchases/recommendations) on the retailer's website was low. This makes sense since most of the recommendations happened during the day, and if the person wanted to get the discount by being the first one to purchase, she had the highest chances when the traffic on the website was the lowest.

There are also other factors that come into play here. Assuming that recommendations are sent to people's personal (non-work) email addresses, then people probably check these email accounts for new email less regularly while at work. So checking personal email while at work and reacting to a recommendation would mean higher chances of getting a discount. Second, there are also network effects, i.e. the more recommendations sent, the higher chance of recommendation collision, the lower 
chance of getting discount, since one competes with the larger set of people.

\section{Recommendations and communities of interest}

Social networks are a product of the contexts that bring people together. The context can be a shared interest in a particular topic or kind of a book. Sometimes there are circumstances, such as a specific job or religious affiliation, that would make people more likely to be interested in the same type of book or DVD. We first apply a community discovery algorithm to automatically detect communities of individuals who exchange recommendations with one another and to identify the kinds of products each community prefers. We then compare the effectiveness of recommendations across book categories, showing that books on different subjects have varying success rates.

\subsection{Communities and purchases}

In aggregating all recommendations between any two individuals in Section 4.1 we showed that the network consists of one large component, containing a little over 100,000 customers, and many smaller components, the largest of which has 634 customers. However, knowing that a hundred thousand customers are linked together in a large network does not reveal whether a product in a particular category is likely to diffuse through it. Consider for example a new science fiction book one would like to market by word-of-mouth. If science fiction fans are scattered throughout the network, with very few recommendations shared between them, then recommendations about the new book are unlikely to diffuse. If on the other hand one finds one or more science fiction communities, where sci-fi fans are close together in the network because they exchange recommendations with one another, then the book recommendation has a chance of spreading by word-of-mouth.

In the following analysis, we use a community finding algorithm CNM04 in order to discover the types of products that link customers and so define a community. The algorithm breaks up the component into parts, such that the modularity Q,

$Q=$ (number of edges within communities) - (expected number of such edges),

is maximized. In other words, the algorithm identifies communities such that individuals within those communities tend to preferentially exchange recommendations with one another.

The results of the community finding analysis, while primarily descriptive, illustrate both the presence of communities whose members are linked by their common interests, and the presence cross-cutting interests between communities. Applying the algorithm to the largest component, we identify many small communities and a few larger ones. The largest contains 21,000 nodes, 5,000 of whom are senders of a relatively modest 335,000 recommendations. More interesting than simply observing the size of communities is discovering what interests bring them together. We identify those interests by observing product categories where the number of recommendations within the community is significantly higher than it is for the overall customer population. Let $p_{c}$ be the proportion of all recommendations that fall within a particular 


\begin{tabular}{r|r|l} 
\# nodes & \# senders & topics \\
\hline 735 & 74 & books: American literature, poetry \\
710 & 179 & sci-fi books, TV series DVDs, alternative rock music \\
667 & 181 & music: dance, indie \\
653 & 121 & discounted DVDs \\
541 & 112 & books: art \& photography, web development, graphical design, sci-fi \\
502 & 104 & books: sci-fi and other \\
388 & 77 & books: Christianity and Catholicism \\
309 & 81 & books: business and investing, computers, Harry Potter \\
192 & 30 & books: parenting, women's health, pregnancy \\
163 & 48 & books: comparative religion, Egypt's history, new age, role playing games
\end{tabular}

Table 5: A sample of the medium sized communities present in the largest component

product category $c$. Then for a set of individuals sending $x_{g}$ recommendations, we would expect by chance that $x_{g} * p_{c} \pm \sqrt{x_{g} * p_{c} *\left(1-p_{c}\right)}$ would fall within category $c$. We note the product categories for which the observed number of recommendations in the community is many standard deviations higher than expected. For example, compared to the background population, the largest community is focused on a wide variety of books and music. In contrast, the second largest community, involving 10,412 individuals (4,205 of whom are sending over 3 million recommendations), is predominantly focused on DVDs from many different genres, with no particular emphasis on anime. The anime community itself emerges as a highly unusual group of 1,874 users who exchanged over 3 million recommendations.

Perhaps the most interesting are the medium sized communities, some of which are listed in Table 5 having between 100 and 1000 members and often reflecting specific interests. Among the hundred or so medium communities, we found, for example, several communities focusing on Christianity. While some of the Christian communities also shared an interest in children's books, broadway musicals, and travel to Italy, others focused on prayer and bibles, still others also enjoyed DVDs of the Simpsons TV series, and others still took an interest in Catholicism, occult spirituality and kabbalah.

Communities were usually centered around a product group, such as books, music, or DVDs, but almost all of them shared recommendations for all types of products. The DVD communities ranged from bargain shoppers purchasing discounted comedy and action DVDs to smaller anime or independent movie communities, to a group of customers purchasing predominantly children's movies. One community focused heavily on indie music, and imported dance and club music. Another seemed to center around intellectual pursuits, including reading books on sociology, politics, artificial intelligence, mathematics, and media culture, listening to classical music and watching neo-noir film. Several communities centered around business and investment books and frequently also recommended books on computing. One business and investment community included fans of the Harry Potter fiction series, while another enjoyed science fiction and adventure DVDs. One of communities with the most particular interests recommended not only business and investing books to one another, but also an unusual number of books on terrorism, bacteriology, and military history. A community of what one can presume are web designers recommended books to one 
another on art and photography, web development, graphical design, and Ray Bradbury's science fiction novels. Several sci-fi TV series such as Buffy the Vampire Slayer and Star Trek appeared prominently in a few communities, while Stephen King and Douglas Clegg featured in a community recommending horror, sci-fi, and thrillers to one another. One community focused predominantly on parenting, women's health and pregnancy, while another recommended a variety of books but especially a collection of cookie baking recipes.

Going back to components in the network that were disconnected from the largest component, we find similar patterns of homophily, the tendency of like to associate with like. Two of the components recommended technical books about medicine, one focused on dance music, while some others predominantly purchased books on business and investing. Given more time, it is quite possible that one of the customers in one of these disconnected components would have received a recommendation from a customer within the largest component, and the two components would have merged. For example, a disconnected component of medical students purchasing medical textbooks might have sent or received a recommendation from the medical community within the largest component. However, the medical community may also become linked to other parts of the network through a different interest of one of its members. At the very least many communities, no matter their focus, will have recommendations for children's books or movies, since children are a focus for a great many people. The community finding algorithm on the other hand is able to break up the larger social network to automatically identify groups of individuals with a particular focus or a set of related interests. Now that we have shown that communities of customers recommend types of products reflecting their interests, we will examine whether these different kinds of products tend to have different success rates in their recommendations.

\subsection{Recommendation effectiveness by book category}

Some contexts result in social ties that are more effective at conducting an action. For example, in small world experiments, where participants attempt to reach a target individual through their chain of acquaintances, profession trumped geography, which in turn was more useful in locating a target than attributes such as religion or hobbies KB78, TM69. In the context of product recommendations, we can ask whether a recommendation for a work of fiction, which may be made by any friend or neighbor, is more or less influential than a recommendation for a technical book, which may be made by a colleague at work or school.

Table 6 shows recommendation trends for all top level book categories by subject. For clarity, we group the results by 4 different category types: fiction, personal/leisure, professional/technical, and nonfiction/other. Fiction encompasses categories such as Sci-Fi and Romance, as well as children's and young adult books. Personal/Leisure encompasses everything from gardening, photography and cooking to health and religion.

First, we compare the relative number of recommendations to reviews posted on the site (column $c_{a v} / r_{p 1}$ of table 6). Surprisingly, we find that the number of people making personal recommendations was only a few times greater than the number of people posting a public review on the website. We observe that fiction books have 


\begin{tabular}{|c|c|c|c|c|c|c|c|c|}
\hline category & $n_{p}$ & $n$ & $c c$ & $r_{p 1}$ & $v_{a v}$ & $\begin{array}{c}c_{a v} / \\
r_{p 1} \\
\end{array}$ & $p_{m}$ & $b * 100$ \\
\hline Books general & 370230 & $2,860,714$ & 1.87 & 5.28 & 4.32 & 1.41 & 14.95 & 3.12 \\
\hline \multicolumn{9}{|l|}{ Fiction } \\
\hline Children & 46,451 & 390,283 & 2.82 & 6.44 & 4.52 & 1.12 & 8.76 & $2.06^{* *}$ \\
\hline Literature & 41,682 & 502,179 & 3.06 & 13.09 & 4.30 & 0.57 & 11.87 & $2.82^{*}$ \\
\hline Mystery & 10,734 & 123,392 & 6.03 & 20.14 & 4.08 & 0.36 & 9.60 & $2.40^{* *}$ \\
\hline Science fiction & 10,008 & 175,168 & 6.17 & 19.90 & 4.15 & 0.64 & 10.39 & $2.34^{* *}$ \\
\hline Romance & 6,317 & 60,902 & 5.65 & 12.81 & 4.17 & 0.52 & 6.99 & $1.78^{* *}$ \\
\hline Teens & 5,857 & 81,260 & 5.72 & 20.52 & 4.36 & 0.41 & 9.56 & $1.94^{* *}$ \\
\hline Comics & 3,565 & 46,564 & 11.70 & 4.76 & 4.36 & 2.03 & 10.47 & $2.30^{*}$ \\
\hline Horror & 2,773 & 48,321 & 9.35 & 21.26 & 4.16 & 0.44 & 9.60 & $1.81^{* *}$ \\
\hline \multicolumn{9}{|l|}{$\overline{\overline{\text { Personal }}}$} \\
\hline Religion & 43,423 & 441,263 & 1.89 & 3.87 & 4.45 & 1.73 & 9.99 & 3.13 \\
\hline Health/Body & 33,751 & 572,704 & 1.54 & 4.34 & 4.41 & 2.39 & 13.96 & 3.04 \\
\hline History & 28,458 & 28,3406 & 2.74 & 4.34 & 4.30 & 1.27 & 18.00 & 2.84 \\
\hline Home/Garden & 19,024 & 180,009 & 2.91 & 1.78 & 4.31 & 3.48 & 15.37 & $2.26^{* *}$ \\
\hline Entertainment & 18,724 & 258,142 & 3.65 & 3.48 & 4.29 & 2.26 & 13.97 & $2.66^{*}$ \\
\hline Arts/Photo & 17,153 & 179,074 & 3.49 & 1.56 & 4.42 & 3.85 & 20.95 & 2.87 \\
\hline Travel & 12,670 & 113,939 & 3.91 & 2.74 & 4.26 & 1.87 & 13.27 & $2.39^{* *}$ \\
\hline Sports & 10,183 & 120,103 & 1.74 & 3.36 & 4.34 & 1.99 & 13.97 & $2.26^{* *}$ \\
\hline Parenting & 8,324 & 182,792 & 0.73 & 4.71 & 4.42 & 2.57 & 11.87 & 2.81 \\
\hline Cooking & 7,655 & 146,522 & 3.02 & 3.14 & 4.45 & 3.49 & 13.97 & $2.38^{*}$ \\
\hline Outdoors & 6,413 & 59,764 & 2.23 & 1.93 & 4.42 & 2.50 & 15.00 & 3.05 \\
\hline \multicolumn{9}{|l|}{ 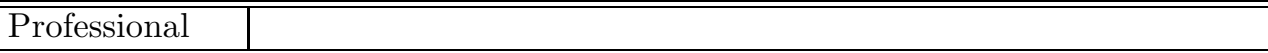 } \\
\hline$\overline{\text { Professional }}$ & 41,794 & 459,889 & 1.72 & 1.91 & 4.30 & 3.22 & 32.50 & $4.54^{* *}$ \\
\hline Business & 29,002 & 476,542 & 1.55 & 3.61 & 4.22 & 2.94 & 20.99 & $3.62^{* *}$ \\
\hline Science & 25,697 & 271,391 & 2.64 & 2.41 & 4.30 & 2.42 & 28.00 & $3.90^{* *}$ \\
\hline Computers & 18,941 & 375,712 & 2.22 & 4.51 & 3.98 & 3.10 & 34.95 & $3.61^{* *}$ \\
\hline Medicine & 16,047 & 175,520 & 1.08 & 1.41 & 4.40 & 4.19 & 39.95 & $5.68^{* *}$ \\
\hline Engineering & 10,312 & 107,255 & 1.30 & 1.43 & 4.14 & 3.85 & 59.95 & $4.10^{* *}$ \\
\hline Law & 5,176 & 53,182 & 2.64 & 1.89 & 4.25 & 2.67 & 24.95 & $3.66^{*}$ \\
\hline \multicolumn{9}{|l|}{ Other } \\
\hline Nonfiction & 55,868 & $\overline{560,552}$ & 2.03 & 3.13 & 4.29 & 1.89 & 18.95 & $3.28^{* *}$ \\
\hline Reference & 26,834 & 371,959 & 1.94 & 2.49 & 4.19 & 3.04 & 17.47 & 3.21 \\
\hline Biographies & 18,233 & 277,356 & 2.80 & 7.65 & 4.34 & 0.90 & 14.00 & 2.96 \\
\hline
\end{tabular}

Table 6: Statistics by book category: $n_{p}$ :number of products in category, $n$ number of customers, $c c$ percentage of customers in the largest connected component, $r_{p 1}$ avg. \# reviews in $2001-2003, r_{p 2}$ avg. \# reviews 1st 6 months 2005, $v_{a v}$ average star rating, $c_{a v}$ average number of people recommending product, $c_{a v} / r_{p 1}$ ratio of recommenders to reviewers, $p_{m}$ median price, $b$ ratio of the number of purchases resulting from a recommendation to the number of recommenders. The symbol ** denotes statistical significance at the 0.01 level, * at the 0.05 level. 
relatively few recommendations compared to the number of reviews, while professional and technical books have more recommendations than reviews. This could reflect several factors. One is that people feel more confident reviewing fiction than technical books. Another is that they hesitate to recommend a work of fiction before reading it themselves, since the recommendation must be made at the point of purchase. Yet another explanation is that the median price of a work of fiction is lower than that of a technical book. This means that the discount received for successfully recommending a mystery novel or thriller is lower and hence people have less incentive to send recommendations.

Next, we measure the per category efficacy of recommendations by observing the ratio of the number of purchases occurring within a week following a recommendation to the number of recommenders for each book subject category (column $b$ of table 6 ). On average, only $2 \%$ of the recommenders of a book received a discount because their recommendation was accepted, and another $1 \%$ made a recommendation that resulted in a purchase, but not a discount. We observe marked differences in the response to recommendation for different categories of books. Fiction in general is not very effectively recommended, with only around $2 \%$ of recommenders succeeding. The efficacy was a bit higher (around 3\%) for non-fiction books dealing with personal and leisure pursuits. Perhaps people generally know what their friends' leisure interests are, or even have gotten to know them through those shared interests. On the other hand they may not know as much about each others' tastes in fiction. Recommendation success is highest in the professional and technical category. Medical books have nearly double the average rate of recommendation acceptance. This could be in part attributed to the higher median price of medical books and technical books in general. As we will see in Section 9.2 a higher product price increases the chance that a recommendation will be accepted.

Recommendations are also more likely to be accepted for certain religious categories: $4.3 \%$ for Christian living and theology and $4.8 \%$ for Bibles. In contrast, books not tied to organized religions, such as ones on the subject of new age $(2.5 \%)$ and occult $(2.2 \%)$ spirituality, have lower recommendation effectiveness. These results raise the interesting possibility that individuals have greater influence over one another in an organized context, for example through a professional contact or a religious one. There are exceptions of course. For example, Japanese anime DVDs have a strong following in the US, and this is reflected in their frequency and success in recommendations. Another example is that of gardening. In general, recommendations for books relating to gardening have only a modest chance of being accepted, which agrees with the individual prerogative that accompanies this hobby. At the same time, orchid cultivation can be a highly organized and social activity, with frequent 'shows' and online communities devoted entirely to orchids. Perhaps because of this, the rate of acceptance of orchid book recommendations is twice as high as those for books on vegetable or tomato growing.

\section{Products and recommendations}

We have examined the properties of the recommendation network in relation to viral marketing. Now we focus on the products themselves and their characteristics that determine the success of recommendations. 


\subsection{How long is the long tail?}

Recently a 'long tail' phenomenon has been observed, where a large fraction of purchases are of relatively obscure items where each of them sells in very low numbers but there are many of those items. On Amazon.com, somewhere between 20 to 40 percent of unit sales fall outside of its top 100,000 ranked products BHS03. Considering that a typical brick and mortar store holds around 100,000 books, this presents a significant share. A streaming-music service streams more tracks outside than inside its top 10,000 tunes Ano05.

We performed a similar experiment using our data. Since we do not have direct sales data we used the number of successful recommendations as a proxy to the number of purchases. Figure 15 plots the distribution of the number of purchases and the number of recommendations per product. Notice that both the number of recommendations and the number of purchases per product follow a heavy-tailed distribution and that the distribution of recommendations has a heavier tail.

Interestingly, figure 15(a) shows that just the top 100 products account for $11.4 \%$ of the all sales (purchases with discount), and the top 1000 products amount to $27 \%$ of total sales through the recommendation system. On the other hand $67 \%$ of the products have only a single purchase and they account for $30 \%$ of all sales. This shows that a significant portion of sales come from products that sell very few times. Recently there has been some debate about the long tail Gom06, And06. Some argue that the presence of the long tail indicates that niche products with low sales are contributing significantly to overall sales online. We also find that the tail is a bit longer than the usual 80-20 rule, with the top $20 \%$ of the products contributing to about half the sales. It is important to note, however, that our observations do not reflect the total sales of the products on the website, since they include only successful recommendations that resulted in a discount. This incorporates both a bias in the kind of product that is likely to be recommended, and in the probability that a recommendation for that kind of product is accepted.

If we look at the distribution in the number of recommendations per product, shown in Figure 15(b), we observe an even more skewed distribution. 30\% of the products have only a single recommendation and the top 56,000 most recommended products (top 10\%) account for $84 \%$ of all recommendations. This is consistent with our previous observations some types of products, e.g. anime DVDs, are more heavily recommended than others.

Next we examine the distribution of the product recommendation success rate. Out of more than half a million products we took all the products with at least a single purchase, of which there are 41,000 (7\%). Figure [16 shows the success rate (purchases/recommendations). Notice that the distribution is not heavy tailed and has a mode at around $1.3 \%$ recommendation success rate. $55 \%$ of the products have a success rate bellow $5 \%$ and there are around $14 \%$ of the products that have a recommendation success rate higher than $20 \%$.

\subsection{Modeling the product recommendation success}

So far we have seen that some products generate many recommendations and some have a better return than others on those recommendations, but one question still remains: what determines the product's viral marketing success? We present a model 


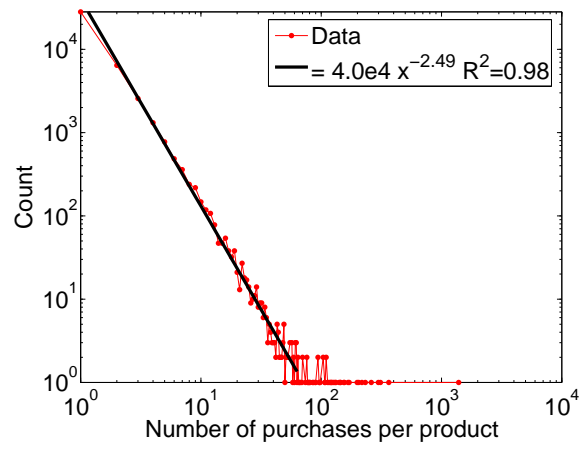

(a) Purchases

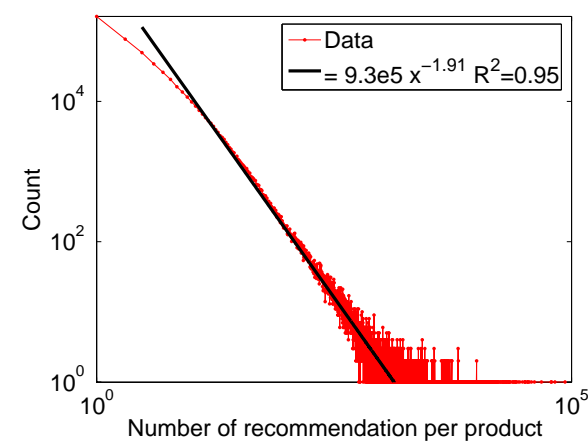

(b) Recommendations

Figure 15: Distribution of number of purchases and recommendations of a product. (a) shows the number of purchases that resulted in a discount per product, and (b) shows the distribution of the number of recommendations per product.

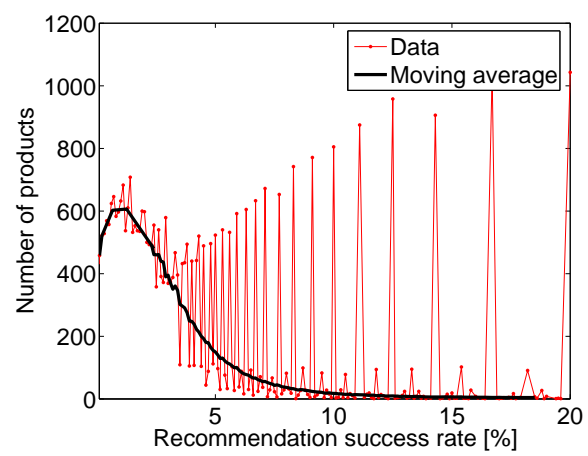

(a) Success rate (linear scale)

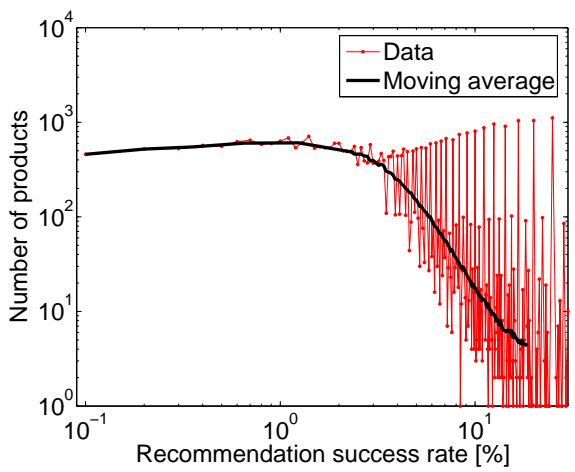

(b) Success rate (log scale)

Figure 16: Distribution of product recommendation success rates. Both plots show the same data: (a) on a linear (lin-lin) scale, and (b) on a logarithmic (log-log) scale. The bold line presents the moving average smoothing.

which characterizes product categories for which recommendations are more likely to be accepted. We use a regression of the following product attributes to correlate them with recommendation success:

- $n$ : number of nodes in the social network (number of unique senders and receivers)

- $n_{s}$ : number of senders of recommendations

- $n_{r}$ : number of recipients of recommendations

- $r$ : number of recommendations 


\begin{tabular}{c|c|c|c|c|c|c|c|c|c}
\hline & $\log (s)$ & $\log (n)$ & $\log \left(n_{s}\right)$ & $\log \left(n_{e}\right)$ & $\log (r)$ & $\log (e)$ & $\log (p)$ & $\log (v)$ & $\log (t)$ \\
\hline $\log (s)$ & 1 & & & & & & & & \\
$\log (n)$ & 0.275 & 1 & & & & & & & \\
$\log \left(n_{s}\right)$ & 0.103 & 0.907 & 1 & & & & & & \\
$\log \left(n_{r}\right)$ & 0.310 & 0.994 & 0.864 & 1.000 & & & & & \\
$\log (r)$ & 0.396 & 0.979 & 0.828 & 0.988 & 1 & & & & \\
$\log (e)$ & 0.392 & 0.981 & 0.831 & 0.990 & 0.999 & 1 & & & \\
$\log (p)$ & 0.185 & 0.098 & 0.088 & 0.098 & 0.107 & 0.106 & 1 & & \\
$\log (v)$ & -0.050 & 0.465 & 0.490 & 0.449 & 0.421 & 0.423 & -0.053 & 1 & \\
$\log (t)$ & -0.031 & 0.064 & 0.071 & 0.061 & 0.056 & 0.056 & -0.019 & 0.269 & 1 \\
\hline
\end{tabular}

Table 7: Pairwise Correlation Matrix of the Books and DVD Product Attributes. $\log (s): \log$ recommendation success rate, $\log (n)$ : $\log$ number of nodes, $\log \left(n_{s}\right)$ : $\log$ number of senders of recommendations, $\log \left(n_{r}\right)$ : $\log$ number of receivers, $\log (r)$ : $\log$ number of recommendations, $\log (e): \log$ number of edges, $\log (p): \log$ price, $\log (v): \log$ number of reviews, $\log (t): \log$ average rating.

- $e$ : number of edges in the social network (number of unique (sender, receiver) pairs)

- $p$ : price of the product

- $v$ : number of reviews of the product

- $t$ : average product rating

From the original set of the half-million products, we compute a success rate $\mathrm{s}$ for the 8,192 DVDs and 50,631 books that had at least 10 recommendation senders and for which a price was given. In section 8.2 we defined recommendation success rate $s$ as the ratio of the total number purchases made through recommendations and the number of senders of the recommendations. We decided to use this kind of normalization, rather than normalizing by the total number of recommendations sent, in order not to penalize communities where a few individuals send out many recommendations (figure 3(b)). Note that in general $s$ could be greater than 1, but in practice this happens extremely rarely (there are only 107 products where $s>1$ which were discarded for the purposes of this analysis).

Since the variables follow a heavy tailed distribution, we use the following model:

$$
s=\exp \left(\sum_{i} \beta_{i} \log \left(x_{i}\right)+\epsilon_{i}\right)
$$

where $x_{i}$ are the product attributes (as described on previous page), and $\epsilon_{i}$ is random error.

We fit the model using least squares and obtain the coefficients $\beta_{i}$ shown in table 8 . With the exception of the average rating, they are all significant, but just the number of recommendations alone accounts for $15 \%$ of the variance (taking all eight variables into consideration yields an R2 of 0.30 for books and 0.81 for DVDs). We should also note that the variables in our model are highly collinear, as can be seen 


\begin{tabular}{c|c|c}
\hline Variable & $\begin{array}{c}\text { Books } \\
\text { Coefficient } \beta_{i}\end{array}$ & $\begin{array}{c}\text { DVD } \\
\text { Coefficient } \beta_{i}\end{array}$ \\
\hline const & $1.317(0.0038)^{* *}$ & $0.929(0.0100)^{* *}$ \\
$n$ & $-0.579(0.0060)^{* *}$ & $0.171(0.0124)^{* *}$ \\
$n_{s}$ & $0.144(0.0018)^{* *}$ & $-0.070(0.0023)^{* *}$ \\
$n_{r}$ & $-0.006(0.0064)$ & $-0.360(0.0104)^{* *}$ \\
$r$ & $0.062(0.0084)^{* *}$ & $-0.002(0.0083)$ \\
$e$ & $0.383(0.0106)^{* *}$ & $0.251(0.0088)^{* *}$ \\
$p$ & $0.013(0.0003)^{* *}$ & $0.007(0.0016)^{* *}$ \\
$v$ & $-0.003(0.0001)^{* *}$ & $-0.003(0.0006)^{* *}$ \\
$t$ & $-0.001(0.0006)^{*}$ & $0.000(0.0009)$ \\
\hline$R^{2}$ & 0.30 & 0.81 \\
\hline
\end{tabular}

Table 8: Regression Using the Log of the Recommendation Success Rate $\log (\mathrm{s})$, as the Dependent Variable for Books and DVDs separately. For each coefficient we provide the standard error and the statistical significance level $\left({ }^{* *}: 0.001,{ }^{*}: 0.1\right)$. We fit separate models for books and DVDs.

from the pairwise correlation matrix (table 7). For example, the number of recommendations $r$ is highly negatively correlated with the dependent variable $(\log (s))$ but in the regression model it exhibits positive influence on the dependent variable. This is probably due to the fact that the number of recommendations is naturally dependent on the number of senders and number of recipients, but it is the high number of recommendations relative to the number of senders that is of importance.

To illustrate the dependencies between the variables we train a Bayesian dependency network Chi03, and show the learned structure for the combined (Books and DVDs) data in figure 17. In this a directed acyclic graph where nodes are variables, and directed edges indicate that the distribution of a child depends on the values taken in the parent variables.

Notice that the average rating $(t)$ is not predictive of the recommendation success rate $(s)$. It is no surprise that the number of recommendations $r$ is predictive of number of senders $n_{s}$. Similarly, the number of edges $e$ is predictive of number of senders $n_{s}$. Interestingly, price $p$ is only related to the number of reviews $v$. Number of recommendations $r$, number of senders $n_{s}$ and price $p$, are directly predictive of the recommendation success rate $s$.

Returning to our regression model, we find that the numbers of nodes and receivers have negative coefficients, showing that successfully recommended products are actually more likely to be not so widely popular. The only attributes with positive coefficients are the number of recommendations $r$, number of edges $e$, and price $p$. This shows that more expensive and more recommended products have a higher success rate. These recommendations should occur between a small number of senders and receivers, which suggests a very dense recommendation network where lots of recommendations are exchanged between a small community of people. These insights could be of use to marketers - personal recommendations are most effective in small, densely connected communities enjoying expensive products. 


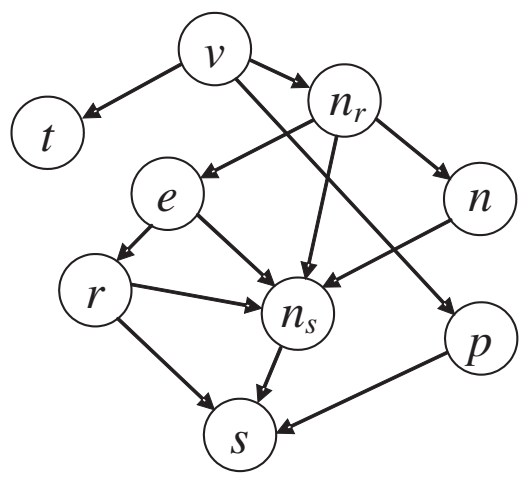

Figure 17: A Bayesian network showing the dependencies between the variables. $s$ : recommendation success rate, $n$ : number of nodes, $n_{s}$ : number of senders of recommendations, $n_{r}: \log$ number of receivers, $r$ : number of recommendations, $e$ : number of edges, $p$ : price, $v$ : number of reviews, $t$ : average rating.

\section{Discussion and Conclusion}

Although the retailer may have hoped to boost its revenues through viral marketing, the additional purchases that resulted from recommendations are just a drop in the bucket of sales that occur through the website. Nevertheless, we were able to obtain a number of interesting insights into how viral marketing works that challenge common assumptions made in epidemic and rumor propagation modeling.

Firstly, it is frequently assumed in epidemic models (e.g., SIRS type of models) that individuals have equal probability of being infected every time they interact AM02, Bai75. Contrary to this we observe that the probability of infection decreases with repeated interaction. Marketers should take heed that providing excessive incentives for customers to recommend products could backfire by weakening the credibility of the very same links they are trying to take advantage of.

Traditional epidemic and innovation diffusion models also often assume that individuals either have a constant probability of 'converting' every time they interact with an infected individual GLM01, or that they convert once the fraction of their contacts who are infected exceeds a threshold Gra78. In both cases, an increasing number of infected contacts results in an increased likelihood of infection. Instead, we find that the probability of purchasing a product increases with the number of recommendations received, but quickly saturates to a constant and relatively low probability. This means individuals are often impervious to the recommendations of their friends, and resist buying items that they do not want.

In network-based epidemic models, extremely highly connected individuals play a very important role. For example, in needle sharing and sexual contact networks these nodes become the "super-spreaders" by infecting a large number of people. But these models assume that a high degree node has as much of a probability of infecting each of its neighbors as a low degree node does. In contrast, we find that there are limits to how influential high degree nodes are in the recommendation network. As a person sends out more and more recommendations past a certain number for a 
product, the success per recommendation declines. This would seem to indicate that individuals have influence over a few of their friends, but not everybody they know.

We also presented a simple stochastic model that allows for the presence of relatively large cascades for a few products, but reflects well the general tendency of recommendation chains to terminate after just a short number of steps. Aggregating such cascades over all the products, we obtain a highly disconnected network, where the largest component grows over time by aggregating typically very small but occasionally fairly large components. We observed that the most popular categories of items recommended within communities in the largest component reflect differing interests between these communities. We presented a model which shows that these smaller and more tightly knit groups tend to be more conducive to viral marketing.

We saw that the characteristics of product reviews and effectiveness of recommendations vary by category and price, with more successful recommendations being made on technical or religious books, which presumably are placed in the social context of a school, workplace or place of worship. A small fraction of the products accounts for a large proportion of the recommendations. Although not quite as extreme in proportions, the number of successful recommendations also varies widely by product. Still, a sizeable portion of successful recommendations were for a product with only one such sale - hinting at a long tail phenomenon.

Since viral marketing was found to be in general not as epidemic as one might have hoped, marketers hoping to develop normative strategies for word-of-mouth advertising should analyze the topology and interests of the social network of their customers. Our study has provided a number of new insights which we hope will have general applicability to marketing strategies and to future models of viral information spread.

\section{ACKNOWLEDGEMENTS}

We thank anonymous reviewers for their insightful comments. This work was partially supported by the National Science Foundation under grants SENSOR-0329549 IIS-0326322 IIS-0534205. This work is also supported in part by the Pennsylvania Infrastructure Technology Alliance (PITA). Additional funding was provided by a generous gift from Hewlett-Packard. Jure Leskovec was partially supported by a Microsoft Research Graduate Fellowship.

\section{References}

[AM02] Roy M. Anderson and Robert M. May. Infectious diseases of humans: Dynamics and control. Oxford Press, 2002.

[And06] C. Anderson. The Long Tail: Why the Future of Business Is Selling Less of More. Hyperion, 2006.

[Ano05] Anonymous. Profiting from obscurity: What the "long tail" means for the economics of e-commerce. Economist, 2005.

[Bai75] Norman Bailey. The Mathematical Theory of Infectious Diseases and its Applications. Griffin, London, 1975. 
[Bas69] Frank Bass. A new product growth for model consumer durables. Management Science, 15(5):215-227, 1969.

[BHS03] Erik Brynjolfsson, Yu Hu, and Michael D. Smith. Consumer surplus in the digital economy: Estimating the value of increased product variety at online booksellers. Management Science, 49(11):1580-1596, 2003.

[BN01] D. Bowman and D. Narayandas. Managing customerinitiated contacts with manufacturers: The impact on share of category requirements and word-of-mouth behavior. Journal of Marketing Research, 38(3):281 297, August 2001.

[BR87] J. J. Brown and P. H. Reingen. Social ties and word-of-mouth referral behavior. The Journal of Consumer Research, 14(3):350-362, 1987.

[Bro98] Po Bronson. Hotmale. Wired Magazine, 6(12), 1998.

[Bur03] Kevin Burke. As consumer attitudes shift, so must marketing strategies. 2003.

[Chi03] David Maxwell Chickering. Optimal structure identification with greedy search. JMLR, 3:507-554, 2003.

[CM05] Damon Centola and Michael Macy. Complex contagion and the weakness of long ties. ftp://hive.soc.cornell.edu/mwm14/webpage/WLT.pdf 2005 .

[CM06] Judith Chevalier and Dina Mayzlin. The effect of word of mouth on sales: Online book reviews. Journal of Marketing Research, 43(3):345, 2006 .

[CNM04] Aaron Clauset, M. E. J. Newman, and Cristopher Moore. Finding community structure in very large networks. Physical Review E, 70:066111, 2004 .

[DL04] Arnaud DeBruyn and Gary Lilien. A multi-stage model of word of mouth through electronic referrals. 2004.

[ER60] P. Erdös and A. Rényi. On the evolution of random graphs. Publ. Math. Inst. Hung. Acad. Sci., 5:17-61, 1960.

[FN93] Jonathan Frenzen and Kent Nakamoto. Structure, cooperation, and the flow of market information. Journal of Consumer Research, 20(3):360375, December 1993.

[GGLNT04] D. Gruhl, R. Guha, D. Liben-Nowell, and A. Tomkins. Information diffusion through blogspace. In $W W W^{\prime} 04,2004$.

[GLM01] J. Goldenberg, B. Libai, and E. Muller. Talk of the network: A complex systems look at the underlying process of word-of-mouth. Marketing Letters, 3(12):211-223, 2001. 
[Gom06] L. Gomes. It may be a long time before the long tail is wagging the web. The Wall Street Jounal, 2006. July 262006.

[Gra73] M. S. Granovetter. The strength of weak ties. American Journal of Sociology, 78:1360-1380, 1973.

[Gra78] M. Granovetter. Threshold models of collective behavior. American Journal of Sociology, 83(6):1420-1443, 1978.

[HN06] Petter Holme and M. E. J. Newman. Nonequilibrium phase transition in the coevolution of networks and opinions. Physical Review E, 74:056108, 2006 .

[HPV06] S. Hill, F. Provost, and C. Volinsky. Network-based marketing: Identifying likely adopters via consumer networks. Statistical Science, 21(2):256 $276,2006$.

[Jur00] S. Jurvetson. What exactly is viral marketing? Red Herring, 78:110-112, 2000.

[KB78] P. Killworth and H. Bernard. Reverse small world experiment. Social Networks, 1:159-192, 1978.

[KKT03] D. Kempe, J. Kleinberg, and E. Tardos. Maximizing the spread of infuence in a social network. In ACM SIGKDD International Conference on Knowledge Discovery and Data Mining (KDD), 2003.

[LAH06] Jure Leskovec, Lada A. Adamic, and Bernardo A. Huberman. The dynamics of viral marketing. In ACM conference on Electronic Commerce, pages 228-237, 2006.

[LSK06] Jure Leskovec, Ajit Singh, and Jon Kleinberg. Patterns of influence in a recommendation network. In Pacific-Asia Conference on Knowledge Discovery and Data Mining (PAKDD), 2006.

[LSY03] G. Linden, B. Smith, and J. York. Amazon.com recommendations: itemto-item collaborative filtering. IEEE Internet Computing, 7(1):76-80, 2003.

[Mon01] Alan L. Montgomery. Applying quantitative marketing techniques to the internet. Interfaces, 30:90-108, 2001.

[RD02] Matt Richardson and Pedro Domingos. Mining knowledge-sharing sites for viral marketing. In $A C M$ SIGKDD International Conference on Knowledge Discovery and Data Mining (KDD), 2002.

[Rog95] Everett M. Rogers. Diffusion of Innovations. Free Press, New York, fourth edition, 1995.

[RZ02] Paul Resnick and Richard Zeckhauser. Trust among strangers in internet transactions: Empirical analysis of ebay's reputation system. In The Economics of the Internet and E-Commerce. Elsevier Science, 2002. 
[SR03] Mani R. Subramani and Balaji Rajagopalan. Knowledge-sharing and influence in online social networks via viral marketing. Communications of the ACM, 46(12):300-307, 2003.

[SS98] David Strang and Sarah A. Soule. Diffusion in organizations and social movements: From hybrid corn to poison pills. Annual Review of Sociology, 24:265-290, 1998.

[TM69] J. Travers and S. Milgram. An experimental study of the small world problem. Sociometry, 32:425-443, 1969.

[Wat02] D.J. Watts. A simple model of global cascades on random networks. PNAS, 99(9):4766-5771, Apr 2002.

[WH04] Fang Wu and Bernardo A. Huberman. Social structure and opinion formation. Computational Economics 0407002, EconWPA, July 2004. available at http://ideas.repec.org/p/wpa/wuwpco/0407002.html.

[YA03] Sha Yang and Greg M. Allenby. Modeling interdependent consumer preferences. Journal of Marketing Research, 40(3):282 - 294, August 2003. 


\section{Appendix}
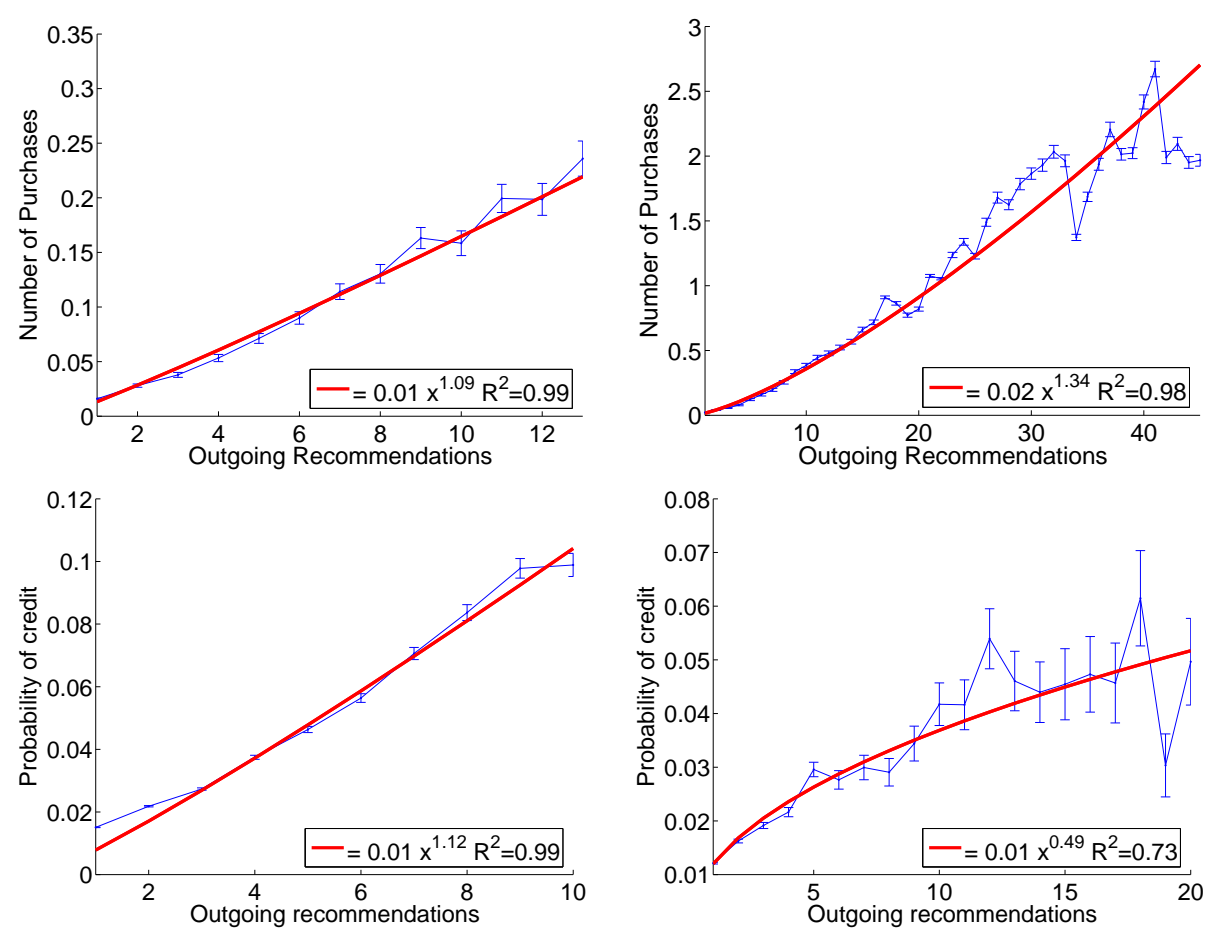

(a) Books

(b) DVD

Figure A-1: Top row: Power fit to the non-linear part of the number of resulting purchases given a number of outgoing recommendations. Bottom row: Power fit to the probability of getting a credit given a number of outgoing recommendations. 


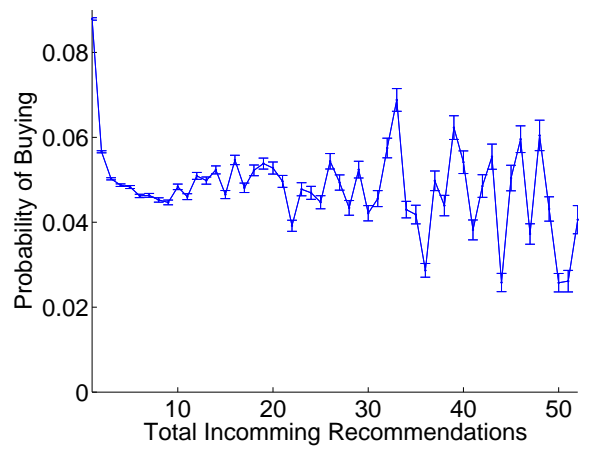

(a) Books

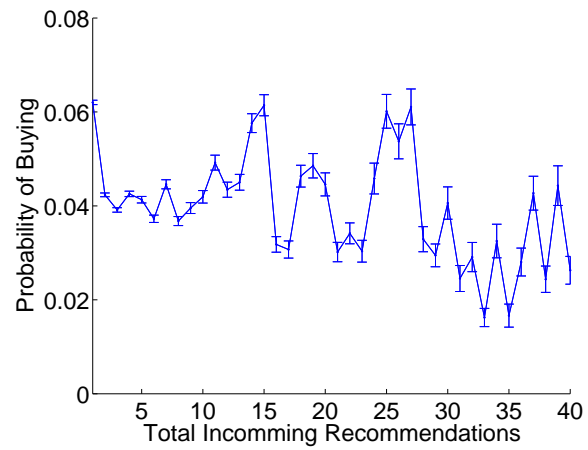

(c) Music

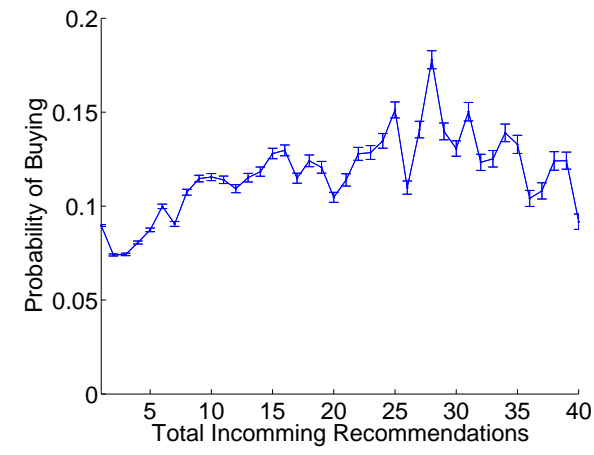

(b) DVD

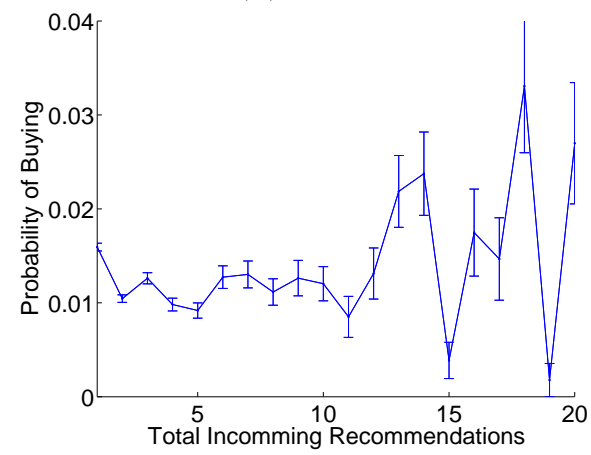

(d) Video

Figure A-2: Probability of buying a product given a total number of incoming recommendations on all products.

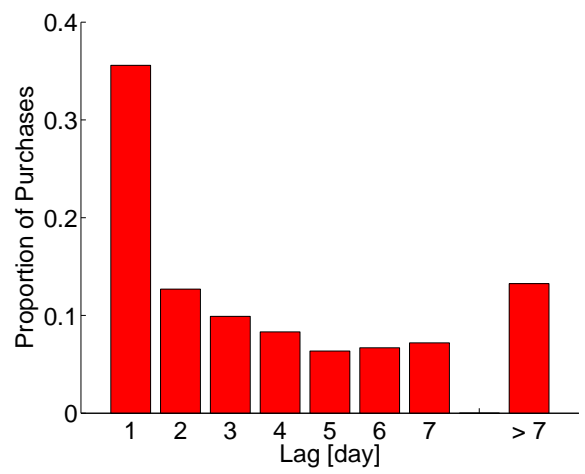

(a) Music

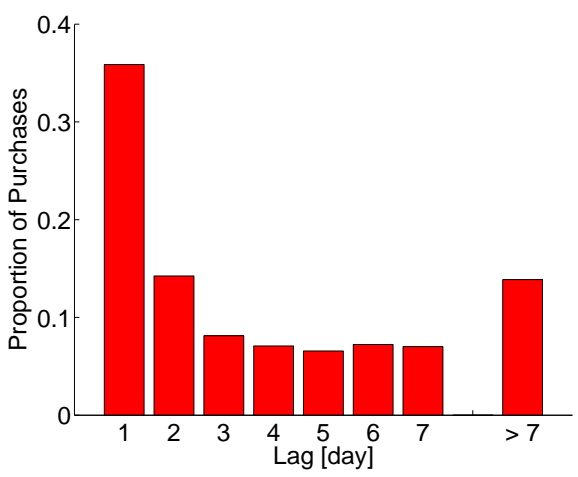

(b) Video

Figure A-3: The time between the first recommendation and the purchase. The histograms show how long does it take to accumulate sufficient number of recommendations to trigger a purchase. Figure 13 plots the same quantity for Books and DVD. The bin size is 1 day. We use all purchases through recommendations. 


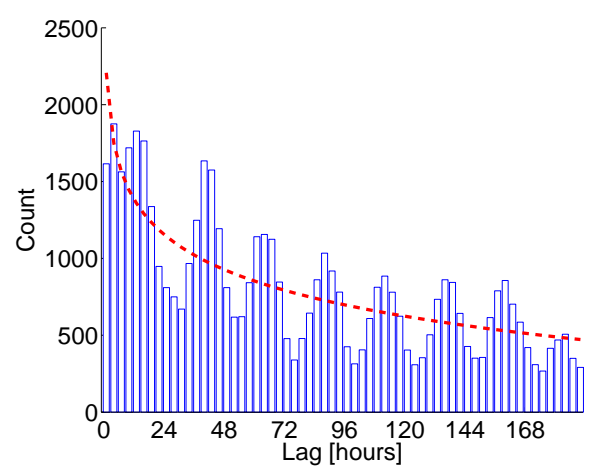

(a) Books

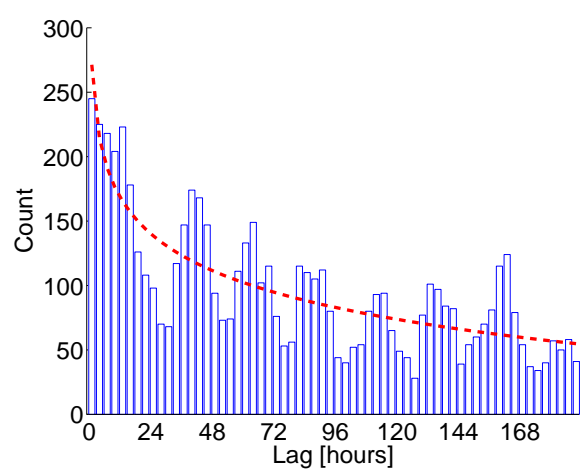

(c) Music

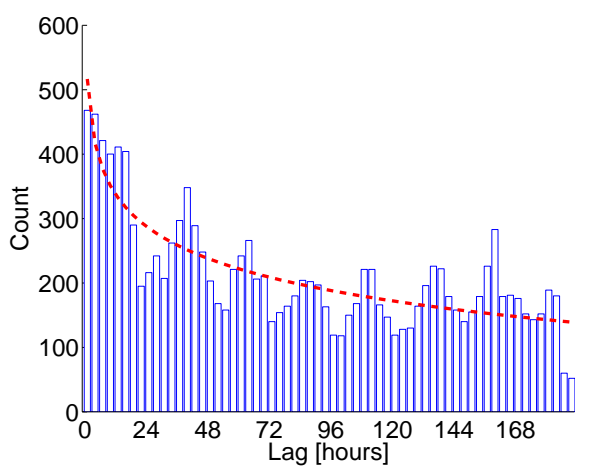

(b) DVD

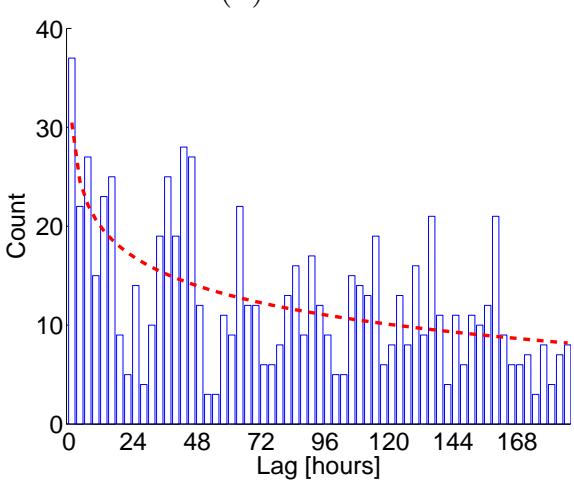

(d) Video

Figure A-4: The time between the recommendation and the purchase taking only the recommendations that resulted in a $10 \%$ discount. The bin size is 3 hours. The dashed line presents a logarithmic fit. 


\begin{tabular}{l|rrrrrrrll}
\hline category & $n_{p}$ & $n$ & $c c$ & $r_{p 1}$ & $r_{p 1} / r_{p 2}$ & $v_{a v}$ & $c_{a v} / r_{p 1}$ & $p_{m}$ & $b$ \\
\hline Anime and Manga & 1301 & 46941 & 18.92 & 14.40 & 17.17 & 4.19 & 2.96 & 26.96 & $28.44^{* *}$ \\
Classics & 266 & 24922 & 25.59 & 9.68 & 6.66 & 4.18 & 4.16 & 22.49 & $11.22^{* *}$ \\
Animation & 237 & 80092 & 11.99 & 41.90 & 19.17 & 4.03 & 3.88 & 22.49 & 10.43 \\
Science Fiction \& Fantasy & 1410 & 317420 & 6.61 & 59.18 & 16.66 & 3.85 & 2.51 & 17.99 & 9.62 \\
Art House \& International & 3185 & 276142 & 7.37 & 24.35 & 12.97 & 3.95 & 2.22 & 22.46 & $9.43^{*}$ \\
Television & 1133 & 195948 & 8.17 & 18.95 & 11.68 & 4.22 & 5.32 & 17.99 & 8.90 \\
Horror & 1125 & 79744 & 13.15 & 30.00 & 9.10 & 3.59 & 1.37 & 17.98 & 8.72 \\
Action and Adventure & 2058 & 248674 & 7.00 & 39.80 & 15.11 & 3.80 & 1.96 & 17.96 & $8.42^{* *}$ \\
Mystery and Suspense & 1683 & 151101 & 9.28 & 26.73 & 10.45 & 3.82 & 2.20 & 17.98 & 7.57 \\
Military and War & 379 & 69180 & 12.53 & 39.31 & 11.14 & 4.12 & 2.26 & 17.96 & 7.41 \\
Cult Movies & 324 & 94049 & 11.24 & 37.93 & 8.45 & 3.89 & 3.34 & 17.98 & 7.28 \\
Kids and Family & 1357 & 230300 & 6.70 & 30.96 & 12.81 & 4.12 & 3.35 & 17.98 & 6.75 \\
Drama & 3376 & 255544 & 7.12 & 25.14 & 11.02 & 3.98 & 2.10 & 17.98 & $6.72^{*}$ \\
Comedy & 2455 & 312033 & 6.08 & 26.25 & 11.14 & 4.02 & 3.30 & 17.98 & $6.01^{* *}$ \\
Musicals \& Performing Arts & 1091 & 88665 & 10.24 & 17.07 & 11.11 & 4.09 & 2.34 & 22.48 & 4.93 \\
Westerns & 234 & 17612 & 24.40 & 11.76 & 7.30 & 3.94 & 2.72 & 13.48 & $4.71^{*}$ \\
Sports & 484 & 23191 & 16.92 & 8.64 & 7.89 & 3.97 & 2.49 & 17.98 & $4.55^{*}$ \\
Documentary & 1058 & 53538 & 15.24 & 6.12 & 9.08 & 3.95 & 3.70 & 17.99 & 4.24 \\
Educational & 89 & 5532 & 19.60 & 3.39 & 2.63 & 3.97 & 5.48 & 19.95 & 3.99 \\
Music Video and Concerts & 2222 & 91657 & 8.44 & 8.06 & 11.16 & 4.09 & 2.88 & 17.99 & 3.85 \\
Special Interests & 963 & 43225 & 10.42 & 5.83 & 7.45 & 3.99 & 3.43 & 18.74 & 2.62 \\
Fitness and Yoga & 223 & 17160 & 2.23 & 14.65 & 6.66 & 3.88 & 2.93 & 17.96 & 1.98 \\
African American Cinema & 81 & 10609 & 17.92 & 16.00 & 9.06 & 4.15 & 3.41 & 17.98 & 1.56
\end{tabular}

Table A-1: Statistics by DVD genre. * denotes significance at the 0.05 level, ${ }^{* *}$ at the 0.01 level 


\begin{tabular}{l|rrrrrrrrr}
\hline category & $n_{p}$ & $n$ & $c c$ & $r_{p 1}$ & $r_{p 1} / r_{p 2}$ & $v_{a v}$ & $c_{a v} / r_{p 1}$ & $p_{m}$ & $b * 100$ \\
\hline Anime and Manga & 962 & 5081 & 9.64 & 13.98 & 18.76 & 4.39 & 0.26 & 17.99 & $1.99^{*}$ \\
Educational & 607 & 6569 & 1.64 & 1.97 & 10.75 & 4.17 & 3.01 & 19.95 & 1.59 \\
Fitness & 920 & 24627 & 0.43 & 8.41 & 12.09 & 4.09 & 1.92 & 14.95 & 1.48 \\
Animation & 171 & 9500 & 4.04 & 61.83 & 19.58 & 4.29 & 0.36 & 17.99 & 1.36 \\
Kids and Family & 4736 & 84608 & 1.13 & 14.26 & 12.11 & 4.29 & 0.85 & 12.98 & 1.16 \\
Special Interests & 3769 & 36862 & 1.45 & 3.19 & 12.73 & 4.14 & 1.65 & 19.95 & 1.09 \\
Mystery and Suspense & 1514 & 13459 & 9.90 & 30.09 & 9.83 & 4.01 & 0.14 & 14.95 & 1.01 \\
Art House \& International & 2459 & 24713 & 3.52 & 17.54 & 10.09 & 4.18 & 0.28 & 17.99 & 0.84 \\
Science Fiction and Fantasy & 1583 & 29565 & 2.54 & 51.92 & 13.76 & 4.01 & 0.18 & 13.99 & 0.83 \\
Documentary & 2936 & 18884 & 1.15 & 3.33 & 9.83 & 4.21 & 0.95 & 19.95 & 0.82 \\
Television & 3632 & 31475 & 0.95 & 5.13 & 12.11 & 4.33 & 1.01 & 14.95 & 0.71 \\
Music Video \& Concerts & 1595 & 14360 & 4.46 & 8.75 & 11.26 & 4.40 & 0.49 & 16.99 & 0.70 \\
Musicals \& Performing Arts & 1621 & 22539 & 3.13 & 13.22 & 9.39 & 4.20 & 0.51 & 19.95 & 0.69 \\
Sports & 1251 & 7987 & 0.49 & 4.07 & 9.83 & 4.15 & 0.91 & 16.99 & 0.69 \\
Comedy & 3645 & 55868 & 2.13 & 22.26 & 10.60 & 4.13 & 0.36 & 13.99 & 0.59 \\
Drama & 4837 & 52691 & 1.87 & 21.72 & 9.25 & 4.15 & 0.26 & 14.95 & 0.56 \\
Military and War & 829 & 10859 & 1.13 & 28.54 & 9.39 & 4.22 & 0.21 & 14.95 & 0.56 \\
Westerns & 487 & 3743 & 1.58 & 9.42 & 6.01 & 4.12 & 0.43 & 9.99 & 0.56 \\
Classics & 326 & 3029 & 0.56 & 8.73 & 8.15 & 4.12 & 0.51 & 14.94 & 0.49 \\
African American Cinema & 87 & 1861 & 0.64 & 15.53 & 7.59 & 4.10 & 0.61 & 9.99 & 0.49 \\
Horror & 935 & 6728 & 1.07 & 36.38 & 9.02 & 3.81 & 0.10 & 12.99 & 0.40 \\
Action and Adventure & 2390 & 25921 & 1.84 & 33.13 & 11.90 & 3.96 & 0.17 & 13.99 & 0.31 \\
Cult Movies & 401 & 5260 & 0.65 & 32.06 & 7.63 & 3.90 & 0.18 & 9.99 & 0.30
\end{tabular}

Table A-2: Statistics for videos in VHS format by genre 


\begin{tabular}{l|rrrrrrrrr}
\hline category & $n_{p}$ & $n$ & $c c$ & $r_{p 1}$ & $r_{p 1} / r_{p 2}$ & $v_{a v}$ & $c_{a v} / r_{p 1}$ & $p_{m}$ & $b$ \\
\hline Broadway and Vocalists & 5423 & 104396 & 4.25 & 6.03 & 13.86 & 4.49 & 1.68 & 14.49 & 2.01 \\
Country & 5876 & 98069 & 4.67 & 5.50 & 18.45 & 4.56 & 1.76 & 13.99 & 1.87 \\
Rock & 10717 & 196852 & 4.10 & 11.00 & 10.18 & 4.40 & 0.99 & 14.99 & 1.87 \\
Alternative Rock & 13405 & 216324 & 5.12 & 13.20 & 11.24 & 4.41 & 0.81 & 13.99 & 1.87 \\
Soundtracks & 4491 & 133507 & 4.81 & 7.92 & 13.82 & 4.38 & 1.77 & 14.99 & 1.87 \\
Classical & 14223 & 116937 & 5.34 & 2.65 & 11.60 & 4.52 & 1.82 & 15.49 & 1.83 \\
Folk & 5244 & 87580 & 5.33 & 4.40 & 13.54 & 4.60 & 2.05 & 14.99 & 1.81 \\
Pop & 16764 & 322431 & 3.30 & 9.55 & 13.19 & 4.43 & 1.22 & 13.99 & 1.78 \\
Opera and Vocal & 5402 & 61643 & 6.08 & 3.32 & 12.90 & 4.48 & 1.69 & 15.99 & 1.73 \\
Miscellaneous & 5823 & 80243 & 5.71 & 3.54 & 12.31 & 4.35 & 1.90 & 13.98 & 1.62 \\
Blues & 2987 & 31199 & 6.62 & 2.76 & 11.53 & 4.59 & 1.89 & 14.99 & 1.54 \\
Hard Rock and Metal & 4787 & 63893 & 4.96 & 18.23 & 7.92 & 4.33 & 0.42 & 14.99 & 1.52 \\
Christian and Gospel & 2977 & 37554 & 2.02 & 5.41 & 16.75 & 4.67 & 1.20 & 14.99 & 1.51 \\
Jazz & 11868 & 113078 & 4.49 & 2.91 & 11.40 & 4.59 & 1.997 & 14.99 & 1.50 \\
Classic Rock & 5711 & 117255 & 4.74 & 13.62 & 6.78 & 4.29 & 0.87 & 13.99 & 1.50 \\
Children s Music & 1755 & 37015 & 4.89 & 3.96 & 12.52 & 4.53 & 2.94 & 12.32 & 1.47 \\
Dance and DJ & 11332 & 139787 & 5.16 & 7.14 & 14.64 & 4.38 & 1.05 & 14.99 & 1.42 \\
New Age & 4219 & 60951 & 5.90 & 3.92 & 13.79 & 4.54 & 1.95 & 14.99 & 1.42 \\
International & 13139 & 130499 & 5.02 & 3.54 & 9.52 & 4.57 & 1.51 & 14.99 & 1.32 \\
Latin Music & 4634 & 38725 & 5.06 & 2.57 & 16.76 & 4.60 & 1.75 & 13.99 & 1.30 \\
Rap and Hip Hop & 3996 & 60135 & 3.67 & 12.23 & 9.64 & 4.38 & 0.67 & 14.99 & 1.14 \\
R\&B & 5965 & 85380 & 2.78 & 8.49 & 12.90 & 4.48 & 0.89 & 13.98 & 1.13
\end{tabular}

Table A-3: Statistics by Music Style 